\title{
The Proceedings of the International Conference
}

on Creationism

Volume 8

Print Reference: Pages 14-35

Article 23

2018

Consistent Young Earth Relativistic Cosmology

Phillip W. Dennis

Unaffiliated

Follow this and additional works at: https://digitalcommons.cedarville.edu/icc_proceedings

Part of the Cosmology, Relativity, and Gravity Commons

DigitalCommons@Cedarville provides a publication platform for fully open access journals, which means that all articles are available on the Internet to all users immediately upon publication. However, the opinions and sentiments expressed by the authors of articles published in our journals do not necessarily indicate the endorsement or reflect the views of DigitalCommons@Cedarville, the Centennial Library, or Cedarville University and its employees. The authors are solely responsible for the content of their work. Please address questions to dc@cedarville.edu.

Browse the contents of this volume of The Proceedings of the International Conference on Creationism.

\section{Recommended Citation}

Dennis, P.W. 2018. Consistent young earth relativistic cosmology. In Proceedings of the Eighth International Conference on Creationism, ed. J.H. Whitmore, pp. 14-35. Pittsburgh, Pennsylvania: Creation Science Fellowship. 


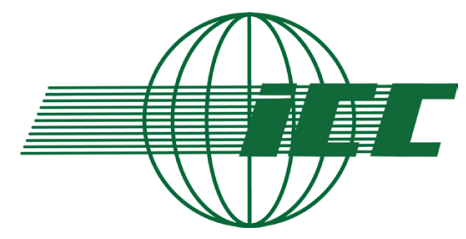

\title{
CONSISTENT YOUNG EARTH RELATIVISTIC COSMOLOGY
}

\author{
Phillip W. Dennis, 1655 Campbell Avenue, Thousand Oaks, California 91360, pwdennis@earthlink.net
}

\section{ABSTRACT}

We present a young earth creationist (YEC) model of creation that is consistent with distant light from distant objects in the cosmos. We discuss the reality of time from theological/philosophical foundations. This results in the rejection of the idealist viewpoint of relativity and the recognition of the reality of the flow of time and the existence of a single cosmological "now." We begin the construction of the YEC cosmology with an examination of the "chronological enigmas" of the inhomogeneous solutions of the Einstein field equations (EFE) of General Relativity (GR). For this analysis we construct an inhomogeneous model by way of the topological method of constructing solutions of the EFE. The topological method uses the local (tensorial) feature of solutions of the EFE that imply that if $(M, g)$ is a solution then removing any closed subset $X$ of $M$ is also a solution on the manifold with $M_{A}=M-X$ and the restriction $g_{A}=\left.g\right|_{M_{A}}$. Also, if $\left(M_{A}, g_{A}\right)$ and $\left(M_{B}, g_{B}\right)$ are solutions of the EFE in disjoint regions then the "stitching" together of $\left(M_{A}, g_{A}\right)$ and $\left(M_{B}, g_{B}\right)$ with continuous boundary conditions is also a solution. From this we show conceptually how an approximate "crude" model with a young earth neighborhood and an older remote universe can be constructed. This approximate "crude" model suffers from having abrupt boundaries. This model is an example of a spherically symmetric inhomogeneous space-time. We discuss the class of exact spherically symmetric inhomogeneous universes represented by the Lemaitre-Tolman (L-T) class of exact solutions of the EFE. A more realistic model refines this technique by excising a past subset with an asymptotically null spacelike surface from the Friedmann-LemaitreRobertson-Walker (FLRW) cosmology. We build the model from the closed FLRW solution by selecting a spacelike hyperboloidal surface as the initial surface at the beginning of the first day of creation. This surface induces, by way of embedding into FLRW space-time, an isotropic but radially inhomogeneous matter density consistent with the full FLRW space-time. The resulting space-time is a subset of the usual FLRW space-time and thus preserves the FLRW causal structure and the observational predictions such as the Hubble law. We show that the initial spacelike surface evolves in a consistent manner and that light from the distant "ancient" galaxies arrives at the earth within the creation week and thereafter. All properties of light arriving from distant galaxies retain the same features as those of the FLRW space-time. This follows from the fact that the solution presented is an open subset of the FLRW space-time so that all differential properties and analysis that applies to FLRW also applies to our solution. Qualitatively these models solve the distant star light problem and from a theological point of view, in which God advances the (cosmic) time of the spacelike hypersurfaces at a non-uniform rate during the miraculous creation week, solve the distant light problem. We conclude by briefly discussing possible objections of some of our key assumptions and showing that a relativist cannot consistently object to our assumptions based on the merely operationalist point of view that an absolute spacelike "now" cannot be empirically determined.

\section{KEY WORDS}

general relativity, young earth cosmology, distant starlight, presentism, $3+1$ formalism

\section{INTRODUCTION}

It is well known that one of the large conundrums of young earth creationist (YEC) models is the cosmological issue of reconciling a large universe with a young earth. Given that the universe is only 6000 years old then no object further than $\sim 6000$ light years would be observable today. A large universe with a uniform global speed of light of $3 \times 10^{8} \mathrm{~m} / \mathrm{s}$ requires a large light transit time from distant objects. Since the current size (diameter) of the observed universe is widely considered to be 92 billion light years, the transit time would seem to require the age of the universe, on the whole, to be in the order of tens of billion years. [Note: Using a radius of 46 Gly for the observable universe and assuming a Minkowski metric (flat universe) yields a light transit time of
46 billion years. The Minkowski result is merely a back of the envelope estimate. However, the universe is not Minkowskian. It is generally known that such a calculation is invalid in general relativistic cosmological models as the curvature and expansion of the universe leads to different results. One less widely recognized effect of expanding space is that space can expand faster than the speed of light while the local speed of light is constant. This explains how the observable size can be greater than the speed of light times the age of the universe. Actual light transit times in GR need to be computed using null geodesics and integrating the time along the geodesic by way of the metric tensor. Such a calculation leads to the usually quoted age of $\sim 13.8$ billion years.] 
Dennis \Young earth relativistic cosmology 2018 ICC

There have been a variety of attempts to solve this discrepancy; these include:

- $\quad$ Tired Light Model (spatial variation of speed of light)

- $\quad$ c-Decay Model (temporal variation of speed of light)

- Pseudophos theory ("false light," light created in transit but not actually emitted from source, hence star image is an illusion)

- "White Hole" models (General Relativity based models)

Of these, the "white hole" models were potentially the most promising as they were based directly on general relativistic physics.

None of these models, as currently developed, however, have adequately solved the starlight issue. They all suffer from either conflict with other observational data, require theologically untenable assumptions, are of an ad hoc nature, or have utilized faulty mathematics/interpretations of metrics and coordinates. Faulkner (2013) discusses the issues with these models (and others). A rigorous and consistent solution is thus still needed. Presented herein is what I believe to be a satisfactory approach to the starlight problem based on inhomogeneous space-times with appropriate relativistic initial conditions. The model relies more on a consistent application and interpretation of a presentist philosophy of time and the relativistic nature of time based on Christian presuppositions rather than on mere technical mathematical details of the several models presented. In fact, I will present one model with an alternative miraculous interpretation of the time aspects of the geometrodynamics during the creation week. In this regard, I agree with Faulkner (2013) when he states we have been: “... thinking primarily in terms of a physical explanation for the light travel time problem, when the solution may be far simpler and more direct" (emph. added). I leave it to the reader to assess whether the proposed solution here is "far simpler and more direct."

It should be emphasized that the models I present are still first approximations; nevertheless, when interpreted properly they do solve the starlight travel problem. More exact models can be developed from the framework presented herein. It is my hope that young earth physicists trained in GR can take the framework presented and produce models with higher fidelity that fit observational data.

To motivate the examination of inhomogeneous models, we note that there has been a long history of physicists stating that the homogeneous cosmological models, e.g. Friedmann-LemaitreRobertson-Walker (FLRW) model are an oversimplification of the physical universe and are best viewed as first order approximate models of the universe. Examples are Dingle (1933) and Tolman (1934). The reader is encouraged to consult Krasiński (1997) for a history of the research on inhomogeneous models.

As more recent observational evidence of large scale structures in the universe has accumulated the study of inhomogeneous models has taken on renewed interest. These recent observations have thereby placed doubt on the "cosmological principle." Examples of such structures include galaxy filaments, "great walls" e.g. the Sloan Great Wall (SGW), superclusters and voids. Several structures larger than the theoretical size limit of 1.2 Gly (see Yadav, 2010) for the cosmological principle have been found. These are (year of discovery in parentheses):

\begin{tabular}{|l|c|}
\hline \multicolumn{1}{|c|}{ Name } & $\begin{array}{c}\text { Size (Giga Light } \\
\text { Years) }\end{array}$ \\
\hline $\begin{array}{l}\text { Hercules-Corona Borealis Great Wall } \\
(2014)\end{array}$ & 10 \\
\hline Giant GRB Ring (2015) & 5.6 \\
\hline Huge-LQG (2012-2013) & 4 \\
\hline U1.11 LQG (2011) & 2 \\
\hline Clowes-Campusano LQG (1991) & 2 \\
\hline Sloan Great Wall (2003) & 1.37 \\
\hline
\end{tabular}

Clowes et al. (2013), in their study of the Huge-LQG, present recent evidences of departures from homogeneity. In particular, they state, "In summary, the Huge-LQG presents an interesting potential challenge to the assumption of homogeneity in the cosmological principle." In addition, Krasiński (1997, p.283) presents the argument that the existence of gravitational lensing implies that the universe cannot be conformally flat. Consequently, he notes that the "universe is not FLRW within the limits set by observation."

In light of such, the homogenous FLRW solutions can only be viewed as first-order local approximations that are useful conceptual tools for interpreting average cosmological effects. It is generally recognized that inhomogeneous models are needed to represent our actual universe. Examples of closed inhomogeneous spherical cosmologies can be found in Zel'dovich (1984) and Sussman (1985). The treatise by Krasiński (1997) is also an invaluable reference.

The outline of this paper is as follows.

(1) We begin with a theological/philosophical discussion of the nature of time. This discussion concludes with the biblically uncontroversial view that time is real and that only the present "now" is real. This view is termed "presentism." This presentist interpretational framework of GR is a major point of this paper.

(2) Having resolved the time issue, we then discuss the theoretical basis of the proposed solution which is the General theory of Relativity (GR). We consider the time development of the standard FLRW cosmological solutions. It is well known that such models predict a lifetime for the universe which is a function of the matter density. This implies that the lifetime of different regions in an inhomogeneous universe will be different. That feature has been the subject of many investigations into structure formation in the universe (such as the large scale inhomogeneities listed above). Within that discussion we explore potential cosmological solutions by examining inhomogeneous cosmologies via the "stitching method." The "stitching method" consists of cutting regions from different solutions of the Einstein field equations (EFE) and "stitching" them together at their boundaries subject to certain continuity conditions. Such models will provide the theoretical framework for the YEC cosmology. 
(3) We then examine general inhomogeneous models. These considerations all point to a solution which is based on the recognition that the EFE depend upon the specification of an initial condition specified on a given initial spatial hypersurface. The general inhomogeneous solution shows that the time of the initial spatial hypersurface is arbitrary within the mathematical framework of GR.

(4) Finally, utilizing the freedom mentioned in (3) to choose the initial creation hypersurface, we examine a cosmological solution using a non-uniform initial density that is constructed by choosing an asymptotically null spacelike initial surface within the FLRW manifold as the initial creation hypersurface at the beginning of day one. We discuss this model and show that it solves the distant light problem.

(5) In closing, we propose that possible future research in YEC cosmologies might benefit from using the $3+1$ formulation of the EFE in which a spacelike initial surface (3-metric $\gamma_{i j}$ and metric 3 -momentum $\pi^{i j}$ ) is integrated forward in time by way of a Hamiltonian approach. The $3+1$ formulation directly corresponds to the presentist philosophy of time, and the initial data can be specified on an initial creation spatial hypersurface and its temporal development examined.

(6) We then summarize our results in the conclusion.

A few words on notation and conventions. Since we will be frequently analyzing spacelike sections of the metric we will use the metric signature $(-,+,+,+)$ for the metric two-form: $d s^{2}=g_{\mu \nu} d x^{\mu} d x^{v}$ Greek indices range over the values $0,1,2,3$. Latin indices are used for the three spatial dimensions and range over the values $1,2,3$. We employ natural units. Newton's gravitational constant $G=1$ and the speed of light $c=1$. Formulae containing masses can be converted to MKS units by replacing a mass $m$ by $\mathrm{Gm} / \mathrm{c}^{2}$.

For convenience of analysis we restrict our attention of solutions with a zero cosmological constant.

Since we will be extensively examining time dependent spacetimes exhibiting spatial isotropy, the metric components in the two-dimensional subspace spanned by $\left(x^{0}, x^{I}\right)$ will be functions only of $x^{0}$ (time) and $x^{l}$ (radial coordinate). To avoid excessive typography, we will regularly employ the following abbreviations

$\dot{R}=\frac{\partial R}{\partial x^{0}} \equiv \frac{\partial R}{\partial t}$

$\stackrel{\text { and }}{R^{\prime}}=\frac{\partial R}{\partial x^{1}} \equiv \frac{\partial R}{\partial r}$

Finally, we will frequently use

$d \Omega^{2}=d \theta^{2}+\sin ^{2} \theta d \varphi^{2}$

for the metric on the two-dimensional unit sphere.

\section{THEOLOGY AND THE PHILOSOPHY OF TIME}

As mentioned in the Introduction, our solution to the starlight and time problem, though fully based on the mathematics of the EFE of GR, will rely essentially on a coherent philosophical and biblically sound interpretational framework for the equations of GR. As such, we begin with a discussion of the nature of time from both the biblical and philosophical perspectives as this is the central framework for the solution to be presented. We will, in particular, examine two philosophies of time called "presentism" and "eternalism." As we shall see, relativity theory does not, in itself, compel one to adopt either of these philosophies. A relativist can be either an eternalist or a presentist. A complete discussion of the philosophy of time cannot be fully addressed in the compass of this article. Those for whom the idea of presentism is new are encouraged to consult the literature on the subject; and in particular see Unger and Smolin (2015), Ellis (2012), Whitrow (1980), and Reichenbach (1956).

The philosophical debate on the nature of time goes back at least to Parmenides and Heraclitus, whose philosophies embody the two modern views of time. For Parmenides unity was absolute and therefore change, along with time, was an illusion; thus, he believed in the unreality of time, or that reality is timeless. Opposed to this view was Heraclitus who held that unity is an illusion and that change is the absolute metaphysical principle. These two views are the perennial opposing philosophical positions on time.

In modern parlance, these two antithetical views are generally referred to as "eternalism" and "presentism."

"Eternalism" is the philosophy that time is an illusion; that past, present and future events (referred to via tensed verbs) are eternally existing in a universe in which time has been "spatialized." It is a universe in which there is no "now" - no unique "present." It is sometimes called a "block-house" universe in which nothing really happens.

"Presentism" is the contrary view that the present is real; that there is an actual real moment called "now," a present moment that continually passes. The past is forever gone, the future will be.

Now fast forward to the $20^{\text {th }}$ century. The philosophy of the nature of time took a dramatic turn in Einstein's theories of relativity.

From that moment scientists and philosophers took up a putative scientific viewpoint of the relativity of time and used it to argue for the unreality of time. Eternalism rose to the ascendency apparently supported by the theories of relativity.

This drift toward a "spatial" view of time and the acceptance of eternalism was, no doubt, encouraged by the mathematical formulation of the theories of relativity in which space-time is conceptualized as a four-dimensional Minkowski space. In Minkowski space, time and space are unified in a four-dimensional manifold ("space-time") with a (local) pseudo-Euclidean "metric" $d s^{2}=d x^{2}+d y^{2}+d z^{2}-c^{2} d t^{2}$.

This presents the notion that all events are locations in a fourdimensional space with coordinates $(x, y, z, t)$ that locate events, and with space-time intervals between events computed via the metric above, much like spatial distances.

It is generally well known that Einstein and Weyl were advocates of the spatialized time of eternalism. Einstein summarized his view as follows (Calaprice, p.75): "For us believing physicists, the distinction between past, present and future is only a stubbornly persistent illusion."

Einstein based his belief upon the impossibility, according to the theory of relativity, of any operational determination of "now," and 
supposedly the lack of a unique objective distinction between past and future, since these apparently depend on the reference frame. As he stated, "The four-dimensional continuum is now no longer resolvable objectively into sections, which contain all simultaneous events; "now" loses for the spatially extended world its objective meaning. It is because of this that space and time must be regarded as a four-dimensional continuum that is objectively unresolvable" (Einstein, 1994, p. 411).

These quotes provide a succinct description of Einstein's philosophy of time.

As Whitrow (1980, p. 4) noted, this viewpoint was concordant with the idealist philosophy. He writes:

"The elimination of time from natural philosophy is closely correlated with the influence of geometry.

... The primary object of Einstein's profound researches on the forces of nature has been well epitomized in the slogan 'the geometrization of physics', time being completely absorbed into the geometry of hyperspace. Thus, instead of ignoring the temporal aspect of nature as Archimedes did, post-renaissance mathematicians and physicists have sought to explain it away in terms of the spatial and in this way they have been aided by philosophers notably the idealists." (emph. added)

As is usual, attempts to analyze time usually smuggle in hidden references to other temporal processes, resulting in viciously circular analysis. Thus, time is "analyzed" in terms of time. For example, Whitrow (1980, p. 348) quotes Weyl (1949, p. 116) 'the objective world simply is, it does not happen. Only to the gaze of my consciousness, crawling upward along the lifeline of my body, does a section of the world come to life as a fleeting image in space which continually changes in time.'

Weyl, as an eternalist, says the objective world does not happen; yet, to make sense of it all, surrenders to a temporal process of crawling along a world line as the generator of change. In this regard, Weyl's view is philosophically incoherent; and his explanation is not philosophically cogent. The problem with the eternalist view, and Weyl in particular, is that by rejecting the reality of the flow of time it replaces a single flow of time with myriads of subjective time flows ("...fleeting image in space which continually changes in time.") in individual consciousnesses crawling along world lines. Hardly a convincing simplification.

So then, under this influence of relativistic physics, time as an illusion was mistakenly embraced by many as a "scientific fact," and the philosophy of "eternalism" was subsequently taken up by philosophers. One of the first, and perhaps more frequently cited papers, is the paper by Hilary Putnam (1995). Putnam's essay outlines and restates the essentials of the arguments of the physicists. However, Putnam's arguments when carefully analyzed are unpersuasive. He commits the usual erroneous interpretations of simultaneity to support the argument and endows the operationalist viewpoint with unwarranted metaphysical conclusions. Appendix A deals with these arguments in detail.

Here we merely point out that the idea that the mathematics of relativity theory compels one to an eternalist view has been correctly denied by many. Originally among these are Eddington and Reichenbach. More recent advocates of the reality of time and the flow of time are Ellis (2012) and Unger and Smolin (2015).

Whitrow (1980, p. 348) commenting on Weyl's remarks above summarizes Eddington's and Reichenbach's criticism of the eternalist philosophy (cf. Figure 1 for the accompanying illustration):

Nevertheless, as has been stressed by Eddington (1935) and Reichenbach (1956 passim) the theory of relativity does not provide a complete account of time. Despite what Weyl has said, the theory is not incompatible with the happening of events but is neutral in this respect. Any given instant $\mathrm{E}$ on the world line of an observer A (who need not be regarded as anything more than recording instrument), all the events from which A can have received signals lie within the backwards-directed light cone with its vertex at E. ... there is an objective time order for all these events and the anomalies of time ordering that Weyl had in mind when he made the statement quoted above concern only events that lie outside this light cone. Signals from these events can only reach A after the event E and when they do reach A they will then lie within A's backwards-directed light cone at that instant. The passage of time corresponds to the continual advance of this light cone. As far as the theory of relativity is concerned, we can consider either the set of all these light cones or the continual transition from one to another. The theory is compatible with either point of view and does not invalidate the concept of temporal transition.

To recap, as Whitrow points out, the pseudo-Euclidean metric of relativity only imposes a causal structure on space-time, and the causal relations are determined by the null cones as shown in Figure 1. The null cones represent the surfaces of fastest causal signals, and thereby separate space-time into regions which can causally influence each other. Within the interior of the forward null cone are future events that can be influenced by event $E$; and within the interior of the backward null cone are the events that can influence $E$. The remainder of space-time, consisting of all events outside the null cone at $E$ (and called spacelike relative to $E$ ), can have no causal connection with $E$. No observer at $E$ can see the spacelike events. In short, an unknown and operationally indeterminable "now" does not imply the nonexistence of "now."

The arguments above answer the eternalist on the basis of their presuppositions -- showing their internal incoherence. However, the strongest argument for the reality of time is from the presupposition of Christian theism.

From a theological perspective, the unreality of time is incompatible with biblical revelation. First, and most important, the reality of time is presented in the Bible in the opening verses of Genesis that describe the miraculous creation week and the occurrence of the first day.

Further, both theologically and philosophically the unreality of time would: (1) imply that all men are co-eternal with God but merely unaware of the fact. This is clearly a version of pantheism; 
(2) entail the unreality of the creation week; (3) imply that the existence of sin, redemption, and judgement are likewise eternal. For instance, Christ is eternally on the cross. These are clearly rejected by the biblical revelation.

So then, fundamentally, presentism is the only biblically consistent position. We cannot, on Christian presuppositions, maintain that the creation is, in anyway, coeternal with God and that all events in salvation history are eternally present. As above, this would mean Christ is forever on the cross and other equally abhorrent implications.

And finally, eternalism is philosophically incoherent. It replaces the mystery of the reality of a single flow of time with billions of subjective flows of time.

To summarize, eternalism is untenable. (1) It is contrary to Scripture. (2) Philosophically it is incoherent and, in fact, selfrefuting. (3) And finally, contrary to some opinion, it is not a consequence of the theory of relativity.

\section{A YEC cosmological solution}

So then, to develop a solution of the starlight problem we turn to inhomogeneous models within GR interpreted according to a biblically consistent presentist philosophy of time. GR is a widely successful theory of gravity and due to the relativity of time ("time dilation") within the theory, GR is recognized as possessing the theoretical framework for solving the time issue. Inhomogeneous models present the possibility of providing different time dilations in different regions of the universe. However, relying on time dilation alone by way of inhomogeneities is not adequate to overcome the large orders of magnitude of the age-to-size ratio. Therefore, we will need to discover another path, in addition to mere inhomogeneity, to solve the light travel time problem. As it turns out, the inhomogeneous solutions contain the seed of the solution since they contain "chronological enigmas" due to issues of the ambiguity of "simultaneity," and the requirement of different lifetimes for different regions of the cosmos.

\section{Foundations of the Solution}

Our solution to the light travel time problem will be based on presentism and the fact that GR specifically and the relativity

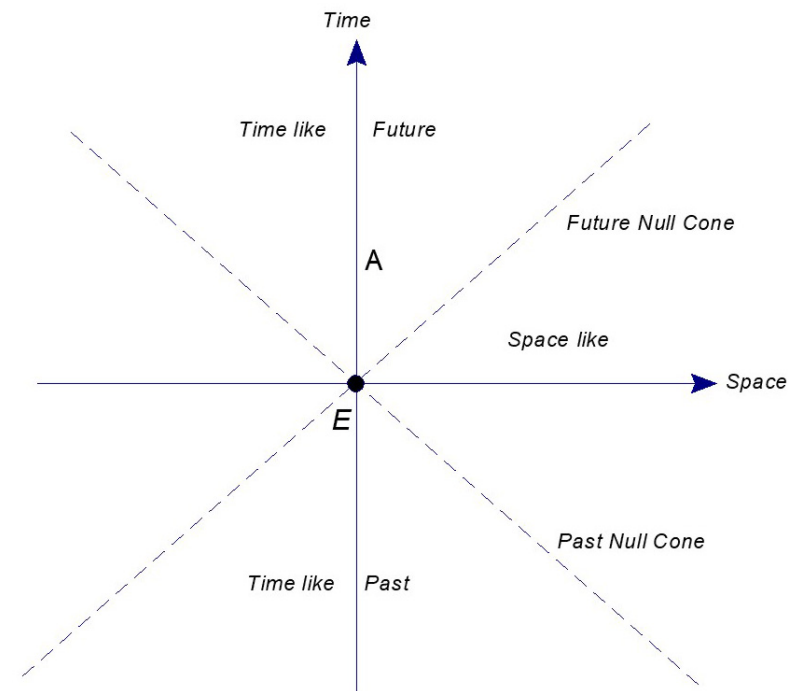

Figure 1. Causal structure of space-time. principle in general prohibits any empirical method of determining a putative hypersurface in space-time that is the present. Thus, any spatial 3-surface that represents an actual "now" (which must exist according to presentism, though in principle operationally undetectable) and explains the distant light arrival is acceptable. As mentioned above, we will construct such a solution motivated by an examination of cosmological solutions with "chronological enigmas" that, when interpreted in the presentist view, imply that there must be "non-simultaneous" (according to a conventional requirement of "cosmic time") yet when interpreted via presentism and a proper selection of a "now" surface accommodate a YEC cosmology. The solutions we will examine are the maximal Schwarzschild geometry and the inhomogeneous L-T models. These cases correspond to time dependent spherically symmetric space-times. We will discuss the Schwarzschild case first then turn to the construction of "crude" inhomogeneous models from the FLRW solution by way of an examination of the general L-T solutions.

To develop an inhomogeneous model cosmology that exhibits "chronological enigmas" we will employ the "cut and stitch" method of assembling solutions from pieces of several cosmological solutions.

\section{The "Cut and Stitch" Approach to GR Solutions}

We will begin the mathematical investigation of a YEC cosmology guided by the topological method of constructing solutions of the Einstein field equations (EFE) of General Relativity (GR).

The object of study in GR is a pseudo-Riemannian manifold denoted by the ordered pair $(M, g)$. Here, $M$ is a $C^{\infty}$ ("smooth") 4-dimensional Hausdorff manifold and $g$ is a Lorentzian metric tensor. We call the ordered pair $(M, g)$ a space-time. See for example, Hawking and Ellis (1973, p. 56-59). A key point made by Hawking and Ellis is that two models denoted by $\left(M^{\prime}, g^{\prime}\right)$ and $\left(M^{\prime \prime}, g^{\prime \prime}\right)$ are equivalent if there exists a diffeomorphism $\theta: M^{\prime} \rightarrow M^{\prime \prime}$ which carries (by way of the differential map, $\theta_{*}$ ) the metric $g^{\prime}$ on $M^{\prime}$ into the metric $g^{\prime \prime}$ on $M^{\prime \prime}$, i.e. $g^{\prime \prime}=\theta_{*} g^{\prime}$. We say that two space-times are locally equivalent (in the regions $\left.N^{\prime}, N^{\prime \prime}\right)$ if for some open subsets $N^{\prime} \subset M^{\prime}$ and $N^{\prime \prime} \subset M^{\prime \prime}$ the space-times $\left(N^{\prime},\left.g^{\prime}\right|_{N^{\prime}}\right)$ and $\left(N^{\prime \prime},\left.g^{\prime \prime}\right|_{N^{\prime \prime}}\right)$ are diffeomorphic. Here $\left.g\right|_{N}$ denotes the restriction of $g$ to the set $N$. It follows that any two locally diffeomorphic space-time manifolds will be physically equivalent in their mutual regions of overlap. We will later use this property to note that our proposed solution, in as much as it matches the FLRW metric, will retrodict all the properties of the FLRW within the common region. An illustration of this can be seen by considering a 2-dimensional example. Consider the unit two-sphere $S^{2}$ embedded in 3-dimensional Euclidean space, $R^{3}$, with induced metric:

$d s^{2}=d \theta^{2}+\sin ^{2} \theta d \varphi^{2}$.

For a second manifold consider the "polar cap" specified by the open set given by

$P=\left\{(\theta, \varphi) \mid \theta<\theta_{0}, 0 \leq \varphi<2 \pi\right\}$.

This is a submanifold of $S^{2}$ and is isomorphic to the same region of $S^{2}$ consequently the polar cap region has all the same local 
geometric properties (for example, curvature, geodesics between points, length of geodesic within the polar region, etc.). This is also the case for the full 4-dimensional case of space-time solutions, the only difference being dimensionality and signature of the metric.

The topological method uses the local (tensorial) feature of solutions of the EFE that imply that if $(M, g)$ is a solution then "cutting" and removing any closed subset $\mathrm{X}$ from $\mathrm{M}$ is also a solution on the manifold $\left(M_{A}, g_{A}\right)$ with $M_{A}=M-X$ and $g_{A}=\left.g\right|_{M_{A}}$. Also, $\operatorname{if}\left(M_{A}, g_{A}\right)$ and $\left(M_{B}, g_{B}\right)$ are two "cut out" solutions of the EFE in disjoint regions then the "stitching" together of $\left(M_{B}, g_{B}\right)$ and $\left(M_{B}, g_{B}\right)$, with continuous boundary conditions is also a solution. We will use this method to show conceptually how an approximate "crude" model with a young earth neighborhood and an older remote universe can be constructed. To construct this model, we will join two regions consisting of different homogeneous densities. Each of these regions is thus a subset of the FLRW cosmology. The two regions will be connected by a vacuum region ("Einstein Rosen bridge") or a "void" consisting of a subset of the Schwarzschild solution. This model is an example of a spherically symmetric inhomogeneous space-time. We will return to the FLRW spacetime below. First, we look at the "chronology enigma" of the vacuum Schwarzschild space-time.

\section{The Schwarzschild Chronology Enigma}

The best known spherically symmetric inhomogeneous solution is the vacuum Schwarzschild metric. Figure 2 shows the maximally extended solution in Kruskal-Szekeres (KS) coordinates (cf. Misner et al., pp. 827-35). (In the figure the coordinates $X, T$ correspond to $u, v$ in Misner et al.). Plotted in the figure are the contours of the Schwarzschild coordinates $(r, t)$ in relation to the KS coordinates $(X, T)$.

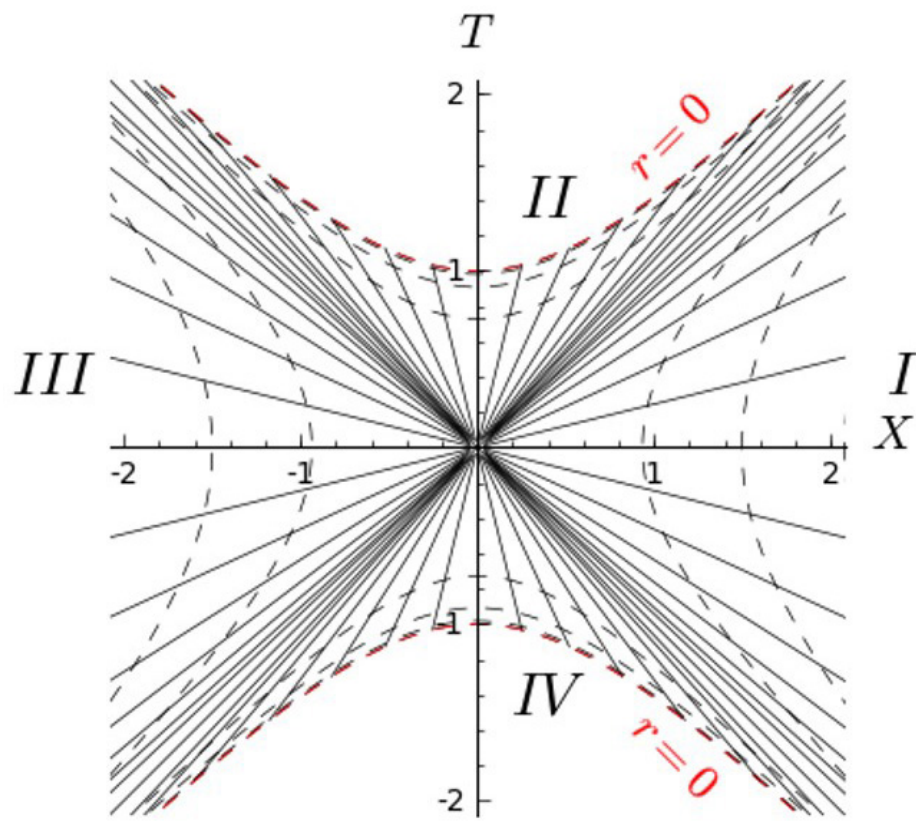

Figure 2. The maximal extension of the Schwarzschild space-time in Kruskal-Szekeres coordinates $(X, T)$. The Schwarzschild curves of constant $r$ are displayed with the broken lines. The $r=0$ singularities are shown. For $T<0$ the singularity is a white hole; while for $T>0$ the singularity is a black hole. The orthogonal solid lines are constant Schwarzschild time coordinates $(t)$.
This solution is sometimes referred to as the "eternal" black hole solution. It is actually a "white hole" at $r=0$ in the past and a "black hole" at $r=0$ in the future. It should be noted that the surfaces $r=0$ are spacelike; so, they are not a place but a time. They do not correspond to the world-line(s) of any physical particle(s) with mass. In this sense, the maximal vacuum Schwarzschild solution is completely devoid of mass, and is an example of pure curvature producing gravitational effects without matter.

The entire manifold consists of four regions labeled in the figure $I, I I, I I I, I V$. These four regions can be characterized as $T$ and $R$ regions according to the criterion whether the gradient of the coordinate of $r$ is timelike (T-region) or spacelike ( $R$-region), cf. Novikov (2001), Frolov and Novikov (1998, pp. 24-5).

A region is said to a T-region if the gradient of $r$ is timelike: $\partial_{\mu} r \partial^{\mu} r<0$

i.e. the normal to the $r=$ constant surface is timelike. In the R-regions the gradient of $r$ is spacelike:

$\partial_{\mu} r \partial^{\mu} r>0$,

i.e. the normal to the $r=$ constant surface is spacelike.

The regions $I I$ and $I V$ are $T$-regions since the gradient of $r$ is timelike there, and thus $r$ is not a time coordinate there. Regions $I$ and $I I I$ are $R$-regions and there, $r$ is a spatial coordinate. \{As an aside, a failure to recognize $T$ and $R$ regions has historically been the source of many misinterpretations of GR solutions.\}

The holes are said to be "eternal" since any observer who maintains a constant radial distance greater than $r=2 M$ (a world line solely in the $R$-region $I$, for example) have world lines that extend from proper time $\tau=-\infty$ to $\tau=\infty$. Such a world line would represent an observer who did not emerge from the past singularity and does not cross the future event horizon (and subsequently falling into the future singularity). On the other hand, any observer freely falling from $r=0$ in region $I V$ to $r=0$ in region $I I$ has a finite temporal history. Such a world line would represent an observer who emerged from the past singularity ("white hole") and crosses the future event horizon falling into the future singularity. This is the source of the temporal enigma. For, if time is real, then the white and black hole must be of finite temporal duration, yet the external region $I$ is of infinite temporal duration. This presents the enigmatic question: "When, relative to the time in the external $R$-region $I$ did the singularities occur?" The time interval between a point on the "white hole" boundary to any point on the "black hole" boundary occurs in finite time. As such those temporally finite world-lines must ultimately be finished relative to the infinite temporal region $I$.

This temporal enigma is akin to the Kantian antinomy (Kant, 1787, A426/B454) that there cannot be an actual infinite past. The argument, in a nut shell, is that the future is a potential infinity which can never be exhausted, yet this KS solution requires not a potential past infinity, but an actualized temporal past infinity consisting of events that have occurred. Time symmetry then implies a contradiction. For Christian theism, we reject out of hand an actualized infinite past since this would entail a "creation" co-eternal with God. Thus, this idealized empty "eternalist" Schwarzschild solution is rejected as theologically and physically 
unacceptable. Rather a solution based on Christian presuppositions requires us to take an initial surface occurring at a finite time in the past. For example, we should take the solution manifold to be the set characterized by:

$M=\left\{(T, X, \theta, \varphi): T>T_{0}\right\}$

for some finite value $T_{0}$ (in general a function of $X, \theta, \varphi$ ) specifying the time of creation.

This is one example of excising an open subset of a solution of EFE. Such an initial condition being an open subset of the KS solution is mathematically consistent with GR.

As it turns out GR provides no single answer to the question of simultaneity and when "in time" the singularities "occur." In fact, GR allows the singularities to "occur" at any causally consistent spacelike surface. This can be illustrated for the case of an external world line that remains forever within the R-region labeled $I$. Referring to Figure 3, cf., for example, Misner et al. (1973, p.528), we display several spacelike surfaces through the Schwarzschild space-time. Any of these could be a surface of simultaneity. The surfaces are temporally ordered: $A A^{\prime}$ ' is earlier than $B B^{\prime}$ ' etc. Each of these surfaces could be taken as an actual surface representing the present "now." And, according to presentism and Christian theism, one such spatial hypersurface must be selected as "now" and, also, there must be an initial hypersurface corresponding to the first moment of creation, since the extension of region $I$ to $t=-\infty$ is inconsistent with Christian theism. As time progresses from each successive "now" ( $A$ to $B$ to $C$ to $D$ ) the proper time on each world line intersecting those surfaces does, of course, progress at different rates, according to the proper time integral. For example, the time registered between space-time events " $A$ " and " $B$ " by any clock (inertial or not) along a world line $\gamma^{a}(\lambda)$ is given by the integral:

$\tau=\int_{A}^{B} \sqrt{g_{\alpha \beta} \frac{d \gamma^{\alpha}(\lambda)}{d \lambda} \frac{d \gamma^{\beta}(\lambda)}{d \lambda}} d \lambda$

Thus, we emphasize, presentism does not deny that local clocks (in particular, non-inertial ones) tick at different rates. Presentism preserves all the differential structure of SR and GR and thus is mathematically consistent with SR and GR.

\section{L-T Chronological Enigmas}

Before analyzing chronology enigmas in general, we now examine the general class of time dependent spherically symmetric solutions of the EFE. These are referred to as the Lemaitre-Tolman (L-T) solutions. These solutions provide the foundation for the analysis of the chronological enigmas. Also, the FLRW cosmologies are a special case of the L-T solutions and can thus be analyzed in terms of the parameters of the L-T class.

\section{A Survey of Spherically Symmetric Inhomogeneous models (L-T Models)}

The general solution for the EFE for an inhomogeneous spherically symmetric space time was developed in detail many years ago (Tolman 1934; Bondi 1947). Frolov and Novikov (1998) give a succinct summary of the process of solving those equations which we follow here with minor changes in notation. Plebański and Krasiński (2006) is also a highly recommended reference with detailed analyses of inhomogeneous cosmological solutions.

The L-T models are based on the time evolution of a spherically symmetric (but otherwise inhomogeneous) dust cloud (no pressure) in comoving coordinates. These are solutions that result from a stress energy tensor that depends only on the mass density and is a function of $t$ and $r$ only:

$T^{\mu v}=\rho(t, r) u^{\mu} u^{v}$

$u^{\mu}$ is the four-velocity vector field of the dust.

It can be shown that the metric interval for the general case of an inhomogeneous spherically symmetric space-time in comoving coordinates of freely falling particles is given by the form:

$d s^{2}=-d t^{2}+g_{r r}(t, r) d r^{2}+R^{2}(t, r) d \Omega^{2}$

The coefficient of $d t^{2}$ is -1 since all clocks are radially free-falling at constant comoving coordinate $r$ and thus register the "cosmic" time $d t^{2}=-d s^{2}$. Note that $R(t, r)$ is no longer a radial coordinate but a function of the comoving coordinate $r$ and the proper time $t$. However, the area of a sphere at time $t$ and radius $r$ is still $4 \pi R^{2}(t, r)$.

The EFE with cosmological constant $\Lambda=0$ and a pressureless "dust" then reduce to the following set of independent equations:

$\frac{1}{2} \dot{R}^{2}-\frac{M(r)}{R}=E(r)$

$g_{r r}(t, r)=\frac{\left(R^{\prime}\right)^{2}}{1+2 E(r)}$

$4 \pi \rho(t, r)=\frac{M^{\prime}(r)}{R^{\prime} R^{2}}$

In these equations: $M(r)$ is gravitational mass within a radius $r$ from the center of symmetry. It is not to be confused with the total invariant rest mass that appears in stress-energy tensor by way of the invariant density $\rho . M(r)$ measures the rest mass energy plus

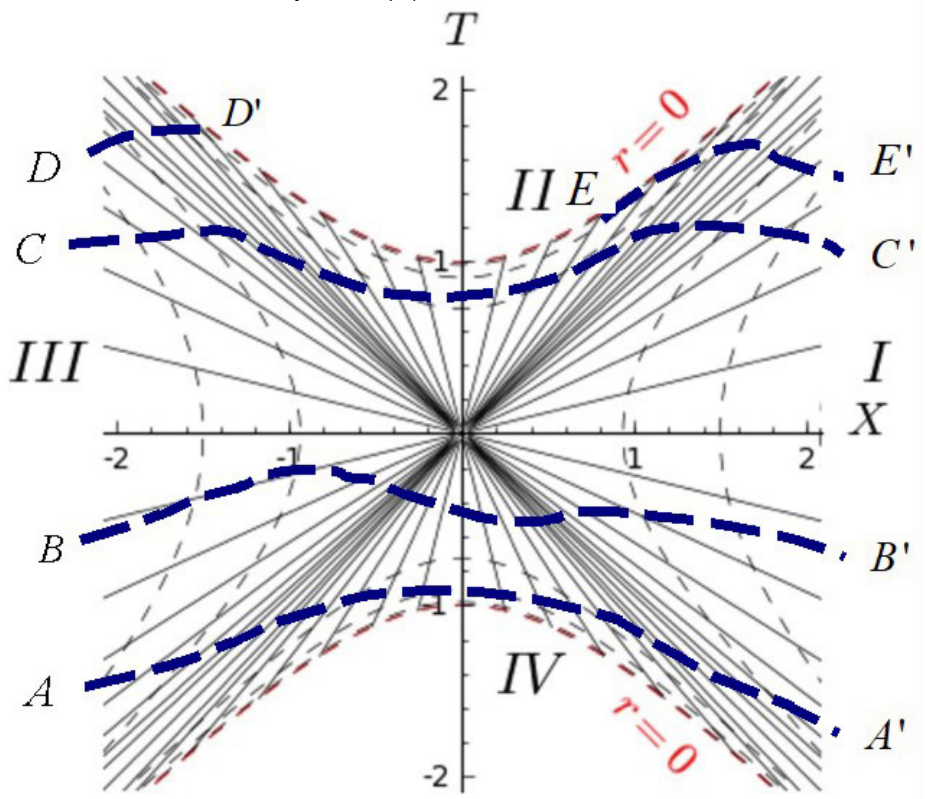

Figure 3. Spacelike hypersurfaces within the maximal extension of the Schwarzschild geometry. 
(a negative) gravitational binding energy. For example, for closed solutions the total gravitational mass can be zero even though $\rho$ (which is always non-negative) is not. $E(r)$ is the energy and curvature at a given comoving radius $r . E(r)$ is required to satisfy the inequality $E(r)>-1 / 2$. For $E(r)<0$ we have a closed universe with positive curvature which expands from an initial "big bang" to a maximum radius then collapses to a final "big crunch." For $E(r)=0$ the universe is open and flat (zero curvature), while for $E(r)>0$ the universe is open and hyperbolic (negative curvature).

An important and interesting feature of these equations is that for fixed $r$ the matter in that "shell" evolves independently from the rest of the matter in the universe that is at a radius $>r$. This is the same as the Newtonian effect that the matter outside a spherical shell does not affect the motion of matter interior to the shell. Each shell of constant $r$ is in fact the equation of a geodesic. Further, each shell can be given its own initial conditions specified by the arbitrary functions $M(r)$ and $E(r)$ and the shell will evolve according to the standard Friedman cosmological model. This observation will play an important part in the YEC solution and its interpretation later. [It should be noted that the functions must satisfy some rather general conditions to avoid surface layers and shell crossings. The details of these conditions are not essential to the overall discussion here. The interested reader is referred to the papers by Hellaby and Lake (1985) or Hellaby (1987) for the details.]

The last equation can be integrated to obtain $M(r)$

$M(r)=\frac{4 \pi}{3} \int_{0}^{r} d r \rho(0, r) \frac{d}{d r}\left[R^{3}(0, r)\right]$

$M(r)$ gives the amount of gravitating mass at radius less than $r$.

In the following we restrict our attention to closed solutions with $-1 / 2<E(r)<0$. For this case, a general solution to equations (4) - (6) can be found by introducing the cycloidal parameter $-\pi \leq \eta \leq \pi$. This choice for $\eta$ corresponds to maximum expansion at $\eta=0$.

$$
\begin{aligned}
& t-t_{B}(r)=\frac{M(r)}{(-2 E(r))^{3 / 2}}(\eta+\sin \eta) \\
& R(t, r)=\frac{M(r)}{(-2 E(r))}(1+\cos \eta)=\frac{M(r)}{(-E(r))} \cos ^{2} \frac{\eta}{2}
\end{aligned}
$$

$t_{B}(r)$ is a constant of integration and an arbitrary function of $r$. In the literature, it is referred to as the local "time to the Big Bang."

Note that this solution is the general inhomogeneous solution for a pressureless dust universe. Another useful representation for this solution can be obtained to express $R(t, r)$ implicitly in terms of $t$ and $r$ is obtained by solving for $\eta$ in the equation for $R(t, r)$

$$
\frac{\eta}{2}=\cos ^{-1}\left[\sqrt{\frac{-E(r) R(t, r)}{M(r)}}\right]
$$

Substituting this into the equation for $t$ then gives the implicit equation for $R$ as a function of $t$ and $r$ :

$$
t-t_{B}(r)=\frac{1}{2 E(r)} \sqrt{2 M(r) R+2 E(r) R^{2}}+\frac{2 M(r)}{(-2 E(r))^{3 / 2}} \cos ^{-1}\left[\sqrt{\frac{-E(r) R}{M(r)}}\right]
$$

The "big bang" surface is given by $R=0$ when $\eta=-\pi$. When we set $\eta=-\pi$ in equation (8) we get for the past singularity:

$t-t_{B}(r)=-\frac{\pi M(r)}{(-2 E(r))^{3 / 2}}$

Since we choose (arbitrarily) $t=0$ as the time of the big bang we obtain:

$t_{B}(r)=\frac{\pi M(r)}{(-2 E(r))^{3 / 2}}$

Thus, explaining the term "time to the big bang."

A special case of the general spherically symmetric solution is the homogeneous FLRW solution. We now briefly discuss the homogeneous FLRW solution since it will play a central role in the conceptual development.

\section{The homogeneous models. The FLRW cosmology}

The FLRW space-time is described by the metric:

$d s^{2}=-d t^{2}+a^{2}(t)\left(d \chi^{2}+f_{K}(\chi) d \Omega^{2}\right)$

$f_{K}(\chi)=\left\{\begin{array}{cc}\sin ^{2} \chi & K=+1 \\ \chi^{2} & K=0 \\ \sinh ^{2} \chi & K=-1\end{array}\right.$

The value of $K$ determines whether the geometry is closed or open. $K=+1$ is the closed solution, $K=0$ is the open conformally flat solution, and $K=-1$ is an open hyperbolic space-time.

The FLRW space-time is the solution for a pressureless and homogeneous "dust" cloud. Recall that this solution uses "comoving" coordinates. The particles are all free falling (only gravity is present), and for any "particle" in the space-time the coordinates $\theta$ and $\varphi$ are constant. We will be using these "comoving" coordinates exclusively in our analysis. In these coordinate systems $t$ is the proper time registered by the freely falling clocks.

The scale factor $a(t)$ can be written in terms of a parametric equation using the cycloidal variable $\eta$.

$a(\eta)=\frac{a_{0}}{2}(1+\cos \eta)$

$t(\eta)=\frac{a_{0}}{2}(\eta+\sin \eta)$

We have chosen the parameter $\eta$ to be in the interval $[-\pi, \pi]$. Here $a_{0}$ is the radius of the universe at maximum expansion corresponding to $\eta=0$. The universe expands from the singularity $a=0$ at $\eta=-\pi$ to the maximum at $\eta=0$. It then collapses to the singularity $a=0$ at $\eta=\pi$. Note that with these choices all particles are synchronized so that they register proper time $t=0$ at maximum expansion.

The radius of maximum expansion and the lifetime of the solution is determined by the matter density $\rho(t)$ in the universe. At 
maximum expansion, the density is $\rho(0)$ and the maximum radius is given by:

$a_{0}=\sqrt{\frac{3}{8 \pi \rho(0)}}$

The lifetime of the universe is then evaluated as:

$$
\begin{aligned}
T & =t(\pi)-t(-\pi) \\
& =\pi a_{0}=\sqrt{\frac{3 \pi}{8 \rho(0)}}
\end{aligned}
$$

\section{Relation of the homogeneous FLRW solution to the L-T solutions}

A special case of the spherically symmetric solutions is the FLRW homogeneous cosmologies which we described above. Here we show the relation of the FLRW solution to the functions $M$ and $E$ of the general L-T solution.

For the FLRW cosmology the matter density is uniform and independent of space, and therefore a function of the comoving time only.

For reference the parameterization of the metric for the FLRW solution is

$d s^{2}=-d t^{2}+a^{2}(t)\left(d \chi^{2}+f_{K}(\chi) d \Omega^{2}\right)$

The general L-T solution as discussed in the section above is given by:

$d s^{2}=-d t^{2}+\frac{\left[R^{\prime}\right]^{2}}{1+2 E} d r^{2}+R^{2} d \Omega$

Comparison with the FLRW metric then yields:

$$
\begin{aligned}
& \frac{\left[R^{\prime}\right]^{2}}{1+2 E}=a^{2}(t) \\
& R^{2}=a^{2}(t) f_{k}(\chi)
\end{aligned}
$$

Therefore

$$
\begin{aligned}
& R^{\prime}=\frac{1}{2} a(t) f_{k}^{-1 / 2}(\chi) f_{k}^{\prime}(\chi) \\
& 2 E(\chi)=\frac{1}{4 f_{k}}\left[f_{k}^{\prime}\right]^{2}-1
\end{aligned}
$$

Specializing to the closed solution we have:

$R^{\prime}=a(t) \cos \chi$

$2 E(\chi)=\cos ^{2} \chi-1=-\sin ^{2} \chi$

With the specialization of the density to a function of time, the functions $E$ and $M$ become (now using $\chi$ as the radial coordinate):

$$
\begin{aligned}
M(\chi) & =\frac{4 \pi}{3} \int_{0}^{\chi} d \chi \rho(0) \frac{d}{d \chi}\left[R^{3}(0, \chi)\right] \\
& =\frac{4 \pi}{3} \rho(0) \int_{0}^{\chi} d \chi \frac{d}{d \chi}\left[R^{3}(0, \chi)\right] \\
& =\frac{4 \pi}{3} \rho(0) R^{3}(0, \chi) \\
& =\frac{4 \pi}{3} \rho(0) a^{3}(0) \sin ^{3} \chi
\end{aligned}
$$

Using the relation given in equation (13) yields:

$M(\chi)=\frac{1}{2} a(0) \sin ^{3} \chi$

We will use these relations to produce a globally inhomogeneous solution (but with piecewise locally homogeneous regions) which will exhibit the features for a YEC cosmology.

\section{A Semi-closed inhomogeneous model}

A few years ago, I attended a presentation in which the speaker presented an inhomogeneous cosmology consisting of two separate regions which are subsets of the FLRW with different total mass. The solution consists of a closed universe consisting of two spherical homogeneous FLRW regions of different uniform density connected by a cylindrical Schwarzschild section with no matter. I will refer to this class of solutions as the "bar bell" cosmologies. Bonnor (1956) also has considered such closed solutions in his investigations of nebulae formation.

If we qualitatively diagram the time dependence of a radial cross section of the space-time we arrive at the notional Figure 4, which depicts the idea of such an inhomogeneous space-time. The left side and right side of the figure depicts regions of homogeneous density. The density of the left side is greater than the density of the region on the right side; hence, the lifetime of the region on the left is less than that of the region on the right by virtue of equation (14). The middle section is a spherically symmetric vacuum (zero matter density) and thus by Birkhoff's theorem must be a section (subset) of the maximal Schwarzschild solution. In that figure, it is evident that one can slide the smaller region's time of existence upward or downward. When one slides the smaller region on the left forward in time we have a cosmology in which the proper "time to the creation" is less than the proper "time to creation" of the larger region. In other words, the EFE do not specify when solutions occur globally. We will need to proceed from this qualitative notional solution by solving the EFE.

Relying on the relativity principle, there is no preferred, i.e. detectable, spacelike surface of "now." If $T\left(x^{0}, x^{1}, x^{2}, x^{3}\right)$ is a scalar function on the space-time such that $\partial_{\mu} T$ is a timelike (i.e. $\left.g^{\mu v} \partial_{\mu} T \partial_{\nu} T<0\right)$ covariant field then $T=$ constant is a physically allowable "now."

This "bar bell" cosmology is an example of a solution obtained by stitching several solutions at the seams. We should note that the "stitching" method requires that the solutions join smoothly along the seams. This condition sometimes rules out many simplistic constructions. [Note: Exact solutions can be obtained provided the stitching condition at the junction hypersurface $f(x)=0$ satisfies 


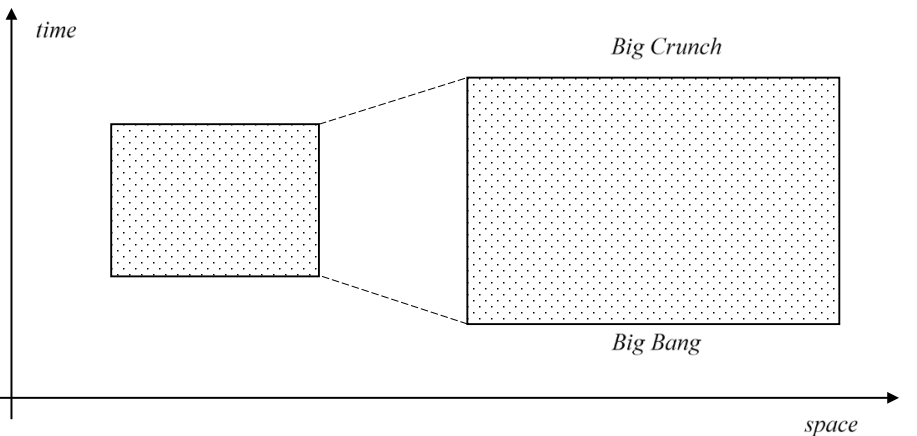

Figure 4. Conceptual diagram of two FLRW regions connected via a vacuum neck. The "Big Bangs" and the "Big Crunches" are nonsimultaneous. Nothing in GR requires that these two regions are necessarily synchronized. Each region can be shifted vertically in time to construct cosmologies with different creation times in the two regions. This model will be analyzed and constructed with exact mathematical solutions of the EFE along with continuous joining conditions in later sections.

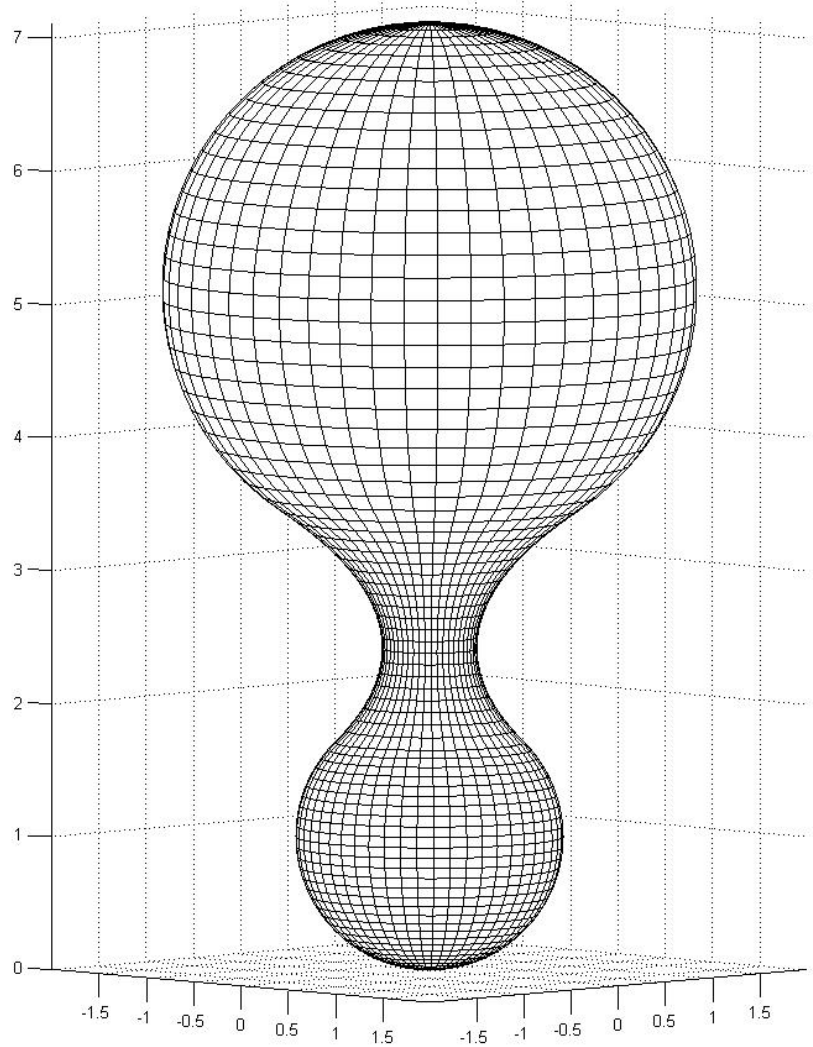

Figure 5. Embedding diagram of the inhomogeneous "bar bell" cosmology. The solution consists of two semi-closed homogeneous FLRW regions which are subsets of the full FLRW cosmological model and a vacuum Schwarzschild region. The FLRW region at the "north pole" (top of the figure) has a smaller matter density at maximal expansion than the FLRW region at the "south pole." The two FLRW regions are connected by an equatorial waist in which the density is zero. The waist is an "EinsteinRosen" bridge and is a subset of the Schwarzschild solution. This diagram is an example of stitching together three solutions of the EFE. The surface of the "bar bell" reflects the geometry of the 2-dimensional cross section of the full space-time at a fixed time and $\theta=\pi / 2$. The coordinates in the figure are $(r, \varphi)$ ( $r$ is measured vertically, and $\varphi$ is axial angle). The independent parameters for this diagram are $\alpha_{1}=3 \pi / 4$ and the maximum radii of the FLRW regions are $a_{1}(0)=a_{2}(0) / 2$. the Lichnerowicz junction conditions (Synge 1971, p.39ff): $\left.G_{\alpha}^{\beta} f_{, \beta}\right|_{+}=\left.G_{\alpha}^{\beta} f_{, \beta}\right|_{-}$ The subscripts + and - indicating evaluation to the "right" and "left" of the two regions to be joined. The differential of $f$ is the normal to the hypersurface $f(x)=0$.]

As mentioned above, an example of straightforward cutting and stitching of solutions is the collapse of an interior homogeneous dust cloud (which is diffeomorphic to a subset of the FLRW cosmology) joined to an exterior vacuum Schwarzschild metric (which is diffeomorphic to a subset of the Kruskal-Szekeres maximal extension of the Schwarzschild solution). See Misner et. al. (1973, pp. 851-3) for the joining in Schwarzschild coordinates. See Novikov (1963), Frolov and Novikov (1998) for the joining in Novikov coordinates which we follow here. The mathematical details of matching the solutions is given in Appendix B. The matching consists of specifying $C^{0}$ (continuous) functions $M(r)$ and $E(r)$ in equations (4) - (6) above. The results are illustrated in Figure 5 and Figure 6.

Figure 5 depicts an embedding diagram of a spatial section of this "bar bell" cosmology at the time of maximum expansion. [Note: the diagram is not a depiction of a potential well. Instead it displays a two-dimensional surface that is the $\theta=\pi / 2$ section of the spatial $S^{3}$ manifold. Each circumference in the diagram is a slice of the two-dimensional sphere, $S^{2}$.] Note the cosmology is closed. It has both a "north" and a "south" pole at the top and bottom of the figure. The spherical sections at the poles are locally homogeneous FLRW regions. The waist in the middle is a section of the Schwarzschild vacuum solution; it is a "void" in the cosmology in which the invariant mass density is zero. This model cosmology exhibits the possible features of general closed inhomogeneous cosmologies.

In Figure 6 we have displayed the temporal evolution of the bar bell cosmology showing the "non-simultaneous big bangs." The "big bang" in the denser "south pole" region occurred later than the "big bang" in the "north pole" region. Also, regions of the Schwarzschild waist come into existence at different times. This solution can be interpreted as two exploding white holes, expanding to a maximum expansion and then collapsing into a black hole. With the cycloidal parametrization above, both FLRW regions have synchronized clocks reading $t=0$ at maximum expansion. The chronology enigma that presents itself is that the "initial" time of each white hole explosion is not necessarily the same (i.e. "simultaneous"). A similar consideration applies to the time of the final collapse to the black hole. Further the total life time of each region is different as the lifetimes of each region are proportional to the mass and inversely proportional to the total energy within each region. As a result, at least one of the events, big bang or big crunch, must be non-simultaneous. The point to be emphasized is that it is not required by GR that any of the singularities be "simultaneous."

\section{A YEC Cosmology Hiding in Plain Sight}

If we look at the general solution to the inhomogeneous cosmology, given in equation (8). above, we notice a rather remarkable feature of the general solution. That feature is the presence of the arbitrary function of integration, $t_{B}(r)$ called there as the "time to the Big Bang." It is this function that allows us to shift the regions in 


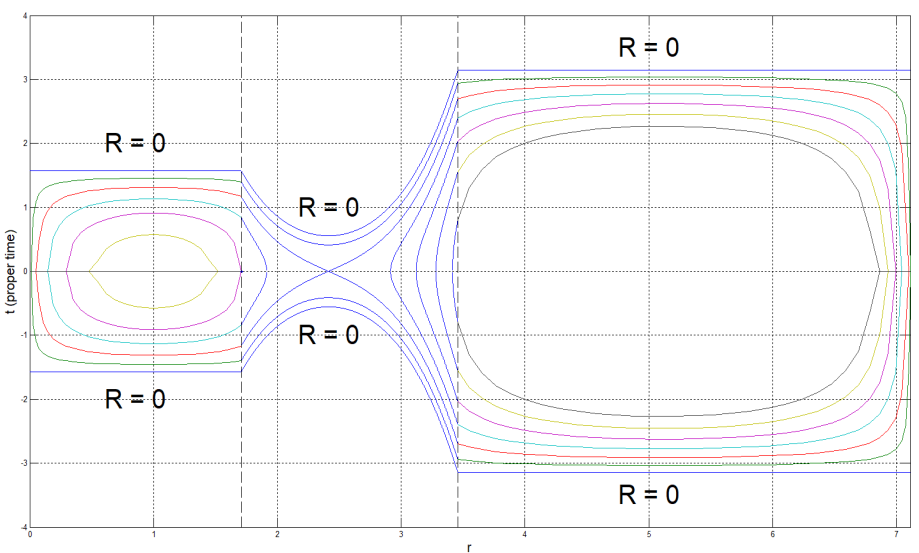

Figure 6. Temporal development of the inhomogeneous "bar bell" cosmology showing the "non-simultaneous big bangs." Proper time is vertical. The "south" pole $(r=0)$ is at the left and the "north" pole at the right. Contours of the "radius" $R$ of the universe are shown in all three regions. The contour interval is $r_{g} / 2=M$. The coordinate system is comoving with clocks synchronized to zero at maximum expansion in the horizontal center line of the graph. Comoving time-like geodesics are specified by a constant value of $r$, and are thus vertical lines in the figure For this choice we see that the "big bang" in the denser "south pole" region occurred later than the "big bang" in the "north pole" region. Also, regions of the Schwarzschild waist come into existence at different times. The vertical dashed lines mark the boundaries between the FLRW regions and the Schwarzschild waist in the middle of the graph. The lines $R=0$ are the boundaries of the space-time. $R=0$ at the bottom of the figure is the "big bang" and $R=0$ at the top of the figure is the "big crunch." Though not labeled in the graph, $R=0$ at the south pole and the north pole.

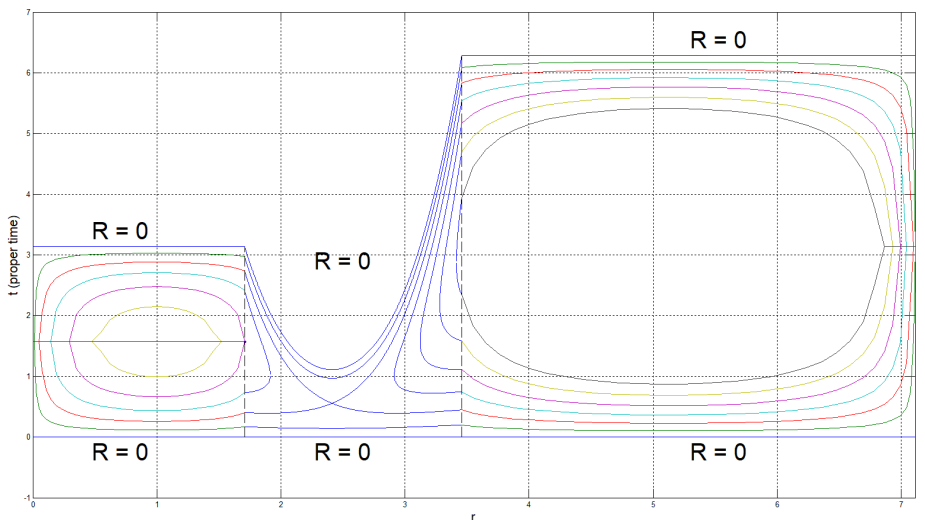

Figure 7. Similar to Figure 6, the temporal development of the inhomogeneous "bar bell" cosmology where the "time to the big bang" $t_{0}(r)$ has been set so that there are "simultaneous big bangs" at time $t=0$, i.e. all clocks are synchronized at the single "big bang." Note that as the new "cosmic time" $t$ advances that the FLRW at the south pole encounters the big crunch before portions of the Schwarzschild waist and the FLRW region at the north pole. The Schwarzschild waist at $r_{0}$ encounters the crunch first. At that time the two FLRW regions become disconnected. All other explanations in Figure 6 apply also.
Figure 4 as mentioned above.

The naturalistic interpretation of the solutions is to extrapolate all the way to $R=0$. As creationists we do not necessarily extrapolate back to $R=0$.

Since the function $t_{B}(r)$ is arbitrary the creationist reply is to choose the freedom in the function $t_{B}(r)$ in a way that it aligns some spacelike hypersurface to correspond with the creation moment. Specifically, we can specify the creation surface via a function $t=t_{C}(r)$. This function depends upon the radial coordinate so that a consistent solution can be achieved by taking a function such that $t-t_{C}(0)$ is only thousands of years, while as the radial coordinate $r$ increases, the time (relative to the comoving "synchronized" time) to the creation surface also increases, reaching values consistent with observational astronomy. In a certain sense this means the earth is young and the distant universe is "old" (relative to the comoving earth clocks). But, as will be discussed later, this does not mean the distant universe has necessarily aged billions of years. As an example of specifying the function $t_{B}(r)$ we can choose the function so that $R=0$ of the "Big Bang" in the "bar bell" cosmology all occur at $t=0$. When we do this, we obtain the cosmology shown in Figure 7.

This feature of "non-simultaneous" Big Bangs in the inhomogeneous models has been noted in the literature. Enqvist (2008), in an analysis of accelerated expansion of the universe, states that, "The universe could have an inhomogeneous big bang, where the universe came into being at different times at different points, and/or an inhomogeneous matter density." (Emphasis added.) This idea is consistent with the YEC model proposed here. We should note that the Enqvist quote does not preclude, due to the relativity of time, the simultaneity of creation though occurring at conceivably different comoving time coordinates. All our theory requires is that the miraculous period of creation occur within a literal week everywhere in the cosmos. Since simultaneity is strictly not determined by time coordinates, we are free to choose any consistent spacelike hypersurface as the simultaneous "now" for the days of the creation week.

To that task we now turn.

\section{A non-simultaneous Big Bang solution}

Motivated by the results of the prior section, let us consider a cosmology with a smooth "creation surface" and smooth initial density. As a model case, we consider the homogeneous FLRW cosmology. The FLRW is given by the metric in equation (11) above.

$$
d s^{2}=-d t^{2}+a^{2}(t)\left(d \chi^{2}+f_{K}(\chi) d \Omega^{2}\right)
$$

As shown in the prior sections GR and the principle of relativity in general allows any spacelike surface to be an acceptable surface for a present "now," and the same applies to the moment of creation. We can exploit the freedom of specifying initial surfaces to select a spacelike surface that would be "old" at remote locations and young near the earth. We are going to cut the "Gordian Knot" of the distant starlight by choosing a spacelike surface that has an "old" universe (in a sense that will become clear) for spatially remote locations, and a young earth. Rejecting the uniformitarian assumption of extrapolating cosmological data to a past creation 
billions of years ago everywhere to a putative initial singularity, we consider extrapolating only thousands of years near the earth and billions of years at remote locations. When we do this, we arrive at the conceptual diagram in Figure 8. This concept produces a surface that bends into the "remote past" (with reference to the usual "cosmic time" of the Big Bang cosmology). Note that if we have the surface approach the past null cone asymptotically, we produce a cosmology in which the light rays will progress toward the earth rapidly as the hyperbolic surface advances in time. We further note that specifying such a surface also produces a spacelike surface with intrinsic curvature differing from the usual FLRW curvature, and a non-uniform matter density (since the density is higher at the remote regions).

In order to select a hypersurface that is asymptotically null, we transform the metric to the conformal form based on the cycloidal variable $\eta$ via equation (12) above:

$d t=a(\eta) d \eta$

Performing the change of time coordinate to the cycloidal parameter yields.

$d s^{2}=a^{2}(\eta)\left[-d \eta^{2}+d \chi^{2}+f_{K}(\chi) d \Omega^{2}\right]$

We take the origin, $\chi=0$, to be in the general vicinity of the earth. Performing the change of time coordinate to the cycloidal parameter yields.

$d s^{2}=a^{2}(\eta)\left[-d \eta^{2}+d \chi^{2}+f_{K}(\chi) d \Omega^{2}\right]$

We note that the past radial $(\dot{\theta}=\dot{\varphi}=0)$ null geodesics are given by the equation:

$-d \eta^{2}+d \chi^{2}=0$

The solution for an incoming null ray is then:

$\chi=-(\eta-\tau)$

In which $\tau$ is the (conformal) time at which the incoming past null ray arrives at $\chi=0$.

We next consider a spacelike hypersurface that is asymptotic to the past null cone. It is given by the equation:

$\eta=\tau-\sqrt{\chi^{2}+b^{2}}$

We consider this to be the creation surface for some value of $\tau$ to be specified. With this choice we are necessarily taking a subset of the usual FLRW, since we are discarding regions of the FLRW manifold for which $\eta \leq \tau-\sqrt{\chi^{2}+b^{2}}$ (cf. Figure 9). Viewed as a cross section of the FLRW cosmology the portion of the FLRW below the surface labeled "Day 1 " did not exist. The constant $b$ is a free parameter of the model. As $b$ approaches zero the spacelike hypersurface approaches the limiting null cone, given by:

$\eta=\tau-\chi$.

Thus, a value of zero would mean the distant light would reach earth instantaneously. For non-zero values $b$ is the time it takes light from objects at infinity to reach the earth.

We now define a new time coordinate by way of the scalar function: $\tau(\eta, \chi)=\eta+\sqrt{\chi^{2}+b^{2}}$

Note that this specification of the function $\tau$ already utilizes a "time to creation" that is a function of $\chi$.

To simplify the analysis, we next introduce a new coordinate $\rho$ via: $\chi=b \sinh \rho$.

Then

$\tau=\eta+b \cosh \rho$

Taking the differentials gives:

$d \eta=d \tau-b \sinh \rho d \rho$

and substituting into the metric we then obtain the following for the metric:

$d s^{2}=a^{2}(\tau-b \cosh \rho)\left[-d \tau^{2}+2 b \sinh \rho d \tau d \rho+b^{2} d \rho^{2}+f_{K}(b \sinh \rho) d \Omega^{2}\right]$

We rewrite the above equation in the following form, for use later, in the $3+1$ formalism:

$d s^{2}=a^{2}(\tau-b \cosh \rho)\left[-d \tau^{2}+b^{2}\left(d \rho+\frac{\sinh \rho}{b} d \tau\right)^{2}-\sinh ^{2} \rho d \tau^{2}+f_{K}(b \sinh \rho) d \Omega^{2}\right]$

$d s^{2}=a^{2}\left[-\cosh ^{2} \rho d \tau^{2}+b^{2}\left(d \rho+\frac{\sinh \rho}{b} d \tau\right)^{2}+f_{K}(b \sinh \rho) d \Omega^{2}\right]$

Note that the actual elapsed proper time registered by the comoving clock at $\rho$ is computed from the conformal time $\tau$ by the integral:

$t=\int a(\tau, \rho) d \tau$

The only difference in the manifold of our cosmology and that of the standard FLRW cosmology is that the initial surface, taken to be the initial creation surface, is a "non-simultaneous" Big Bang relative to the usual FLRW "cosmological time" but viewed as simultaneous within the hyperbolic surface. If from that moment time advances uniformly then the asymptotically null spacelike surfaces maintain their hyperbolic property. However, there is nothing to preclude God from advancing the remote regions more rapidly thereby yielding a non-null hyper-surface. That concept is consistent with the biblical account. Figure 9 illustrates this concept.

Relativistic principles do not distinguish any preferred initial geometry or any preferred cosmological simultaneity surface. Hence, the hyperbolic surface though not "simultaneous" with the $\tau=0$ surface of the FLRW cosmology is no less physically plausible. The illusion is only due to viewing the YEC as embedded in the maximally extended FLRW manifold.

We can plot the paths of radial light rays using the conformal metric in above. Light rays are determined by setting $d s^{2}=0$. This gives:

$-\cosh ^{2} \rho d \tau^{2}+b^{2}\left(d \rho+\frac{\sinh \rho}{b} d \tau\right)^{2}=0$

Or,

$\cosh \rho d \tau= \pm b\left(d \rho+\frac{\sinh \rho}{b} d \tau\right)$

Solving this differential equation yields, the following for incoming and outgoing null rays:

$\tau-\tau_{0}=\left\{\begin{array}{l}b\left(\mathrm{e}^{\rho}-\mathrm{e}^{\rho_{0}}\right) \text { for outgoing light rays } \\ b\left(\mathrm{e}^{-\rho}-\mathrm{e}^{-\rho_{0}}\right) \text { for incoming light rays }\end{array}\right.$

Figure 10 shows the light rays for our YEC cosmology. We note that in Figure 10 the space-time is displayed with the $\tau=0$ creation 
surface as a horizontal line rather than as a hyperboloid embedded in the FLRW manifold. That instantaneous surface is, of course, a curved manifold. As discussed above GR places no temporal constraints on whether any spacelike surfaces are excluded. In fact, relativity forbids that man can operationally determine what is the current "now" surface. This is the case because we can only observe the universe along the past null cone.

We can use equation (21) to compute the particle horizon which gives the observable universe at a given time. For an incoming light ray emitted from $\rho_{0}$ at the creation time $\tau_{0}=0$ and arriving at the earth $(\rho=0)$ at time $\tau$ the solution for the horizon $\rho_{0}$ is:

$\rho_{0}=-\log \left(1-\frac{\tau}{b}\right)$

This shows that the entire universe is observable after the passage of time $b$.

In particular, we note that in this model the light from distant objects arrives at the young earth. Further that all observable physics will be in line with the equations of GR and the implications of the FLRW metric (or any modifications of that solution for inhomogeneities) since the solution is in fact a section of the FLRW manifold. All tensorial equations and invariant quantities are the same. In particular, the predictions of red-shifts are identical to those of the FLRW cosmology.

\section{Postscript. Future Avenues of Research. The $3+1$ Formalism.} Ihad initially intended to analyze the entire class of YEC cosmologies by utilizing the $3+1$ formulation of EFE. Unfortunately, due to time constraints, I have not been able to pursue that approach to the depth and rigor required for publication, at this time. I propose that possible future research in YEC cosmologies might benefit from using the $3+1$ formulation of the EFE in which a spacelike initial surface (3-metric $\gamma_{i j}$ and metric 3-momentum $\pi^{i j}$ ) is integrated forward in time by way of a Hamiltonian approach. The 3+1 formulation directly corresponds to the presentist philosophy of time, and the initial data, namely.

To analyze the time development of solutions to EFE, the $3+1$ formalism decomposes space-time into a foliation of spatial hypersurfaces indexed by time $t$. Each hypersurface $\Sigma_{t}$ can thus be interpreted as a surface of "now;" with the time coordinate $t$ denoting the actual cosmic time. In this way the $3+1$ formalism conceptually reflects the presentist view of time. The 3-dimensional metric $\gamma_{i j}$ and its momentum $\pi^{i j}$ can be specified on an initial creation spatial hypersurface and its temporal development examined. Details of the $3+1$ formalism can be found in detail in Gourgoulhon (2012). The metric is written in terms of the intrinsic metric $\gamma_{i j}$ of the

\section{Time}

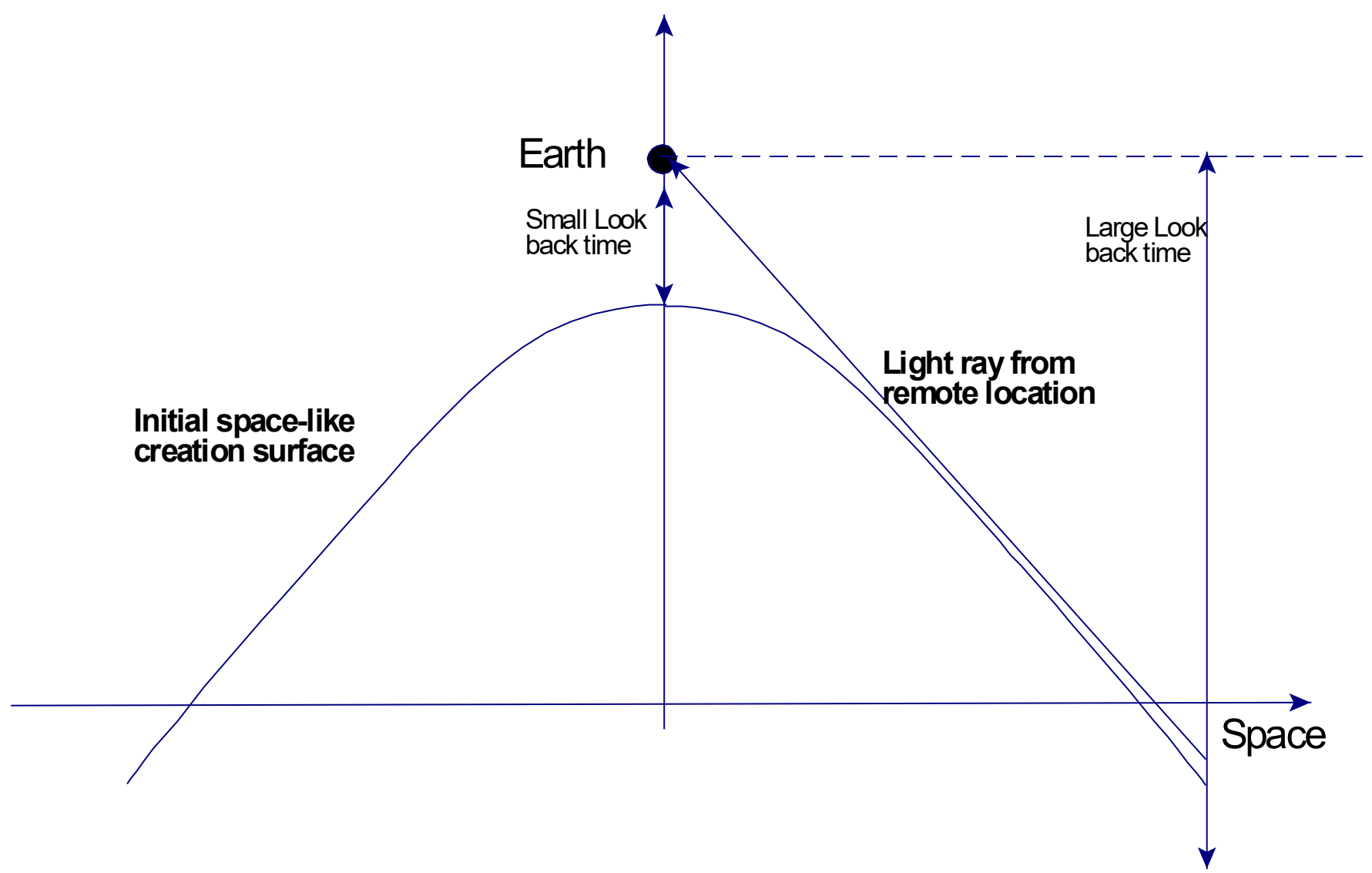

Figure 8. A solution to the light travel time problem based on a hyperbolic initial creation surface given by a function $t=t_{C}(r)$ in terms of the FLRW cosmic time coordinate. Due to the curvature of the surface the look-back time at the earth increases with distance. The creation surface occurs simultaneously at the beginning of day one, even though the "look-back time" (relative to the FLRW extrapolated "cosmic time") for distant events is very large. The look-back time will be a smooth function of distance in the detailed model. 
three-dimensional hypersurface $\Sigma_{t}$ along with the lapse and shift Metric of $\Sigma_{t}$ :

functions, $N$ and $\beta^{j}$ :

$$
d s^{2}=-N^{2} d t^{2}+\gamma_{i j}\left(d x^{i}+\beta^{i} d t\right)\left(d x^{j}+\beta^{j} d t\right)
$$

When equation (22) is compared with equation (20) above one obtains, the following:

$\gamma=b^{2} a^{2}(\eta) d \rho \otimes d \rho+a^{2}(\eta) f_{K}(b \sinh \rho) d \Omega^{2}$

Lapse function $N$ :

$$
\begin{aligned}
N(\tau, \rho) & =a(\tau-b \cosh \rho) \cosh \rho \\
& =a(\eta) \cosh \rho
\end{aligned}
$$

The EFE rewritten in terms of these quantities are (cf. Gourgoulhon, 2012, p.87):

Shift vector $\beta$ :

$$
\begin{aligned}
& \left(\frac{\partial}{\partial t}-L_{\beta}\right) \gamma_{i j}=-2 N K_{i j} \\
& \left(\frac{\partial}{\partial t}-L_{\beta}\right) K_{i j}=-D_{i} D_{j} N+N\left\{R_{i j}+K K_{i j}-2 K_{i k} K_{j}^{k}+4 \pi\left[(S-E) \gamma_{i j}-2 S_{i j}\right]\right\}
\end{aligned}
$$

$\beta^{j}=\left(\frac{\sinh \rho}{b}, 0,0\right)$

$$
R+K^{2}-K_{i j} K^{i j}=16 \pi E
$$

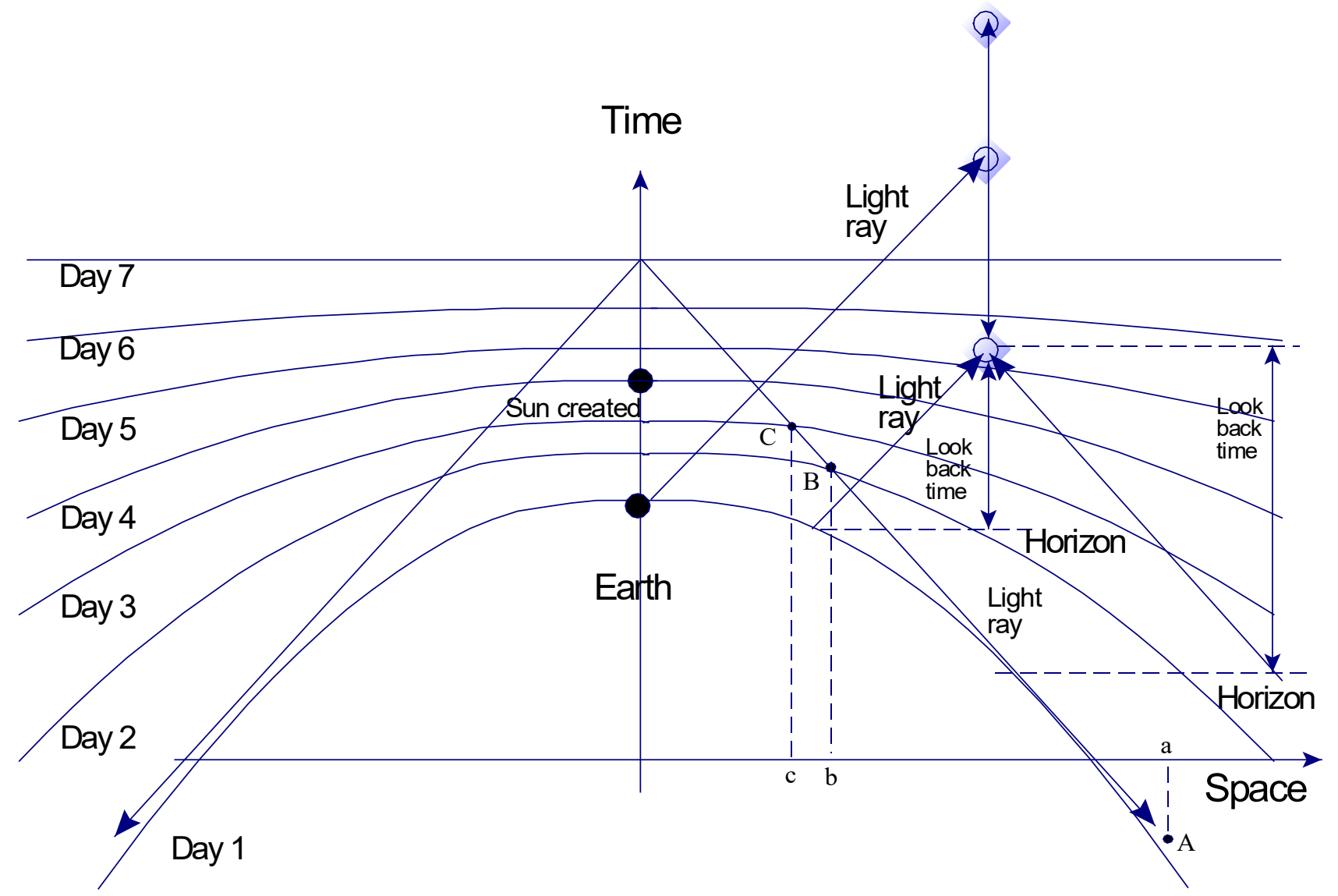

Figure 9. Possible development of the past spacelike surfaces during the creation week in which time is miraculously advanced more rapidly at distant locations resulting in an unfolding of the hyperboloidal sheet as time advances. However, there is nothing fundamentally objectionable to the initial surface propagating in time in a way such that the hyperbolic surface is maintained. If the figure is viewed as a cross section of the FLRW cosmology then we note that the portion of the FLRW below the surface labeled "Day 1" did not exist. As remarked in the text, this solution is thus a subset of the usual full FLRW cosmology ("Big bang cosmology"). The surface labeled "Day 1" is to be viewed as the original miraculous creation of space-time and the stretching out of the heavens. If one visualizes the hyperbolic curves advancing up the time axis it is easy to note that the distant light, though emitted with large look-back times according to the usual FLRW time coordinate, arrives at the earth within a lapsed time of mere days. For example, since the light ray from "A" to "B" lies entirely within the "sandwich" bounded by surfaces labeled Day 1 and Day 2, the event labeled "A," though potentially a vast distance away, has a light ray that arrives at "B" within 1 day. Note, by the projections of a, b, c of A, B, and C onto the space axis, that light traveled a greater distance, $\overline{a b}$, on Day 1 than the distance, $\overline{b c}$, on Day 2 . Note the figure is not to scale and the curvature of the hyperbolic surface is specifiable by the parameter $b$ in the model. As $b$ approaches zero the hyperbolic surfaces approach the null-cone. Regardless, it should be noted that, as drawn, the entire cosmos (in fact, objects at possibly infinite distances and look back times) is visible at the earth on Day 7. 
$D_{j} K_{i}^{j}-D_{i} K=8 \pi p_{i}$

$L_{\beta} \gamma_{i j}=D_{i} \beta_{j}+D_{j} \beta_{i}$

$L_{\beta}$ denotes the Lie derivative with respect to the vector field $\beta$.

$K_{i j}$ is the extrinsic curvature of the surface $\Sigma$ given by $K_{i j}=-\gamma_{i}^{\alpha} \gamma_{j}^{\beta} \nabla_{\alpha} n_{\beta}$ where $\nabla_{\alpha}$ is the four-dimensional covariant derivative. $n_{\beta}$ is the 1 -form field normal to surface $t=$ constant, and $\gamma_{\beta}^{\alpha}=\delta_{\beta}^{\alpha}+n^{\alpha} n_{\beta}$ is the projector onto the $t=$ constant hypersurfaces.

$K=\gamma^{i j} K_{i j}$

$R_{i j}$ is the Ricci tensor for the surface $\sum$ and $R=\gamma^{i j} R_{i j}$ is the Ricci scalar.

The following variables are the decomposition of the stress-energy tensor $T^{\mu v}$ in terms of the $3+1$ splitting. $S_{i j}$ is the spatial part of the stress-energy tensor. $S=\gamma^{i j} S_{i j}$ is its trace.

$E$ is the energy density

$p_{i}$ is the momentum density.

Of course, the YEC version of the FLRW given above in equation (20), being a solution of the EFE, automatically satisfies the $3+1$ equations. The initial condition, specified by our creation surface, was constructed from the implied embedding of the hypersurface in the FLRW cosmology, and it trivially satisfies the dynamic 3+1 equations. This 3 -metric subsequently propagates in time in a way that maintains its curvature. This is expected since the momentum $\pi^{i j}$ of the metric was constrained from the embedding within the FLRW thus determining the extrinsic curvature $K_{i j}$ in equation (23) above and elsewhere. More interesting cases will result from specifying different values for $K_{i j}$ along an initial creation surface.

\section{CONCLUSION}

We have presented a consistent YEC cosmological model that satisfies the EFE and reproduces the observational consequences of both the FLRW space-time ("big bang") and any modifications such as inhomogeneities. The model only differs from conventional solutions in that it uses an initial condition ("creation hypersurface") that supports a young earth. There can be no unprejudiced objection to this solution. A prejudiced objection would rest only upon the assumption of requiring a naturalistic metaphysics requiring, in some sense, a synchronized (and simultaneous) explosion of all matter from a white hole by extrapolating a current value of the metric backward in a presumed preferred time to a putative initial singularity. Such an assumption is not dictated by GR. In fact, we have seen that GR allows for solutions with regions of differing "life times" and "non-simultaneous" (according to a conventional time coordinate) big bangs. This feature of "non-simultaneous" big bangs includes the external Schwarzschild solutions for collapsing stars and the class of general inhomogeneous L-T cosmologies. As we remarked, this flexibility in the theory allows us to choose an

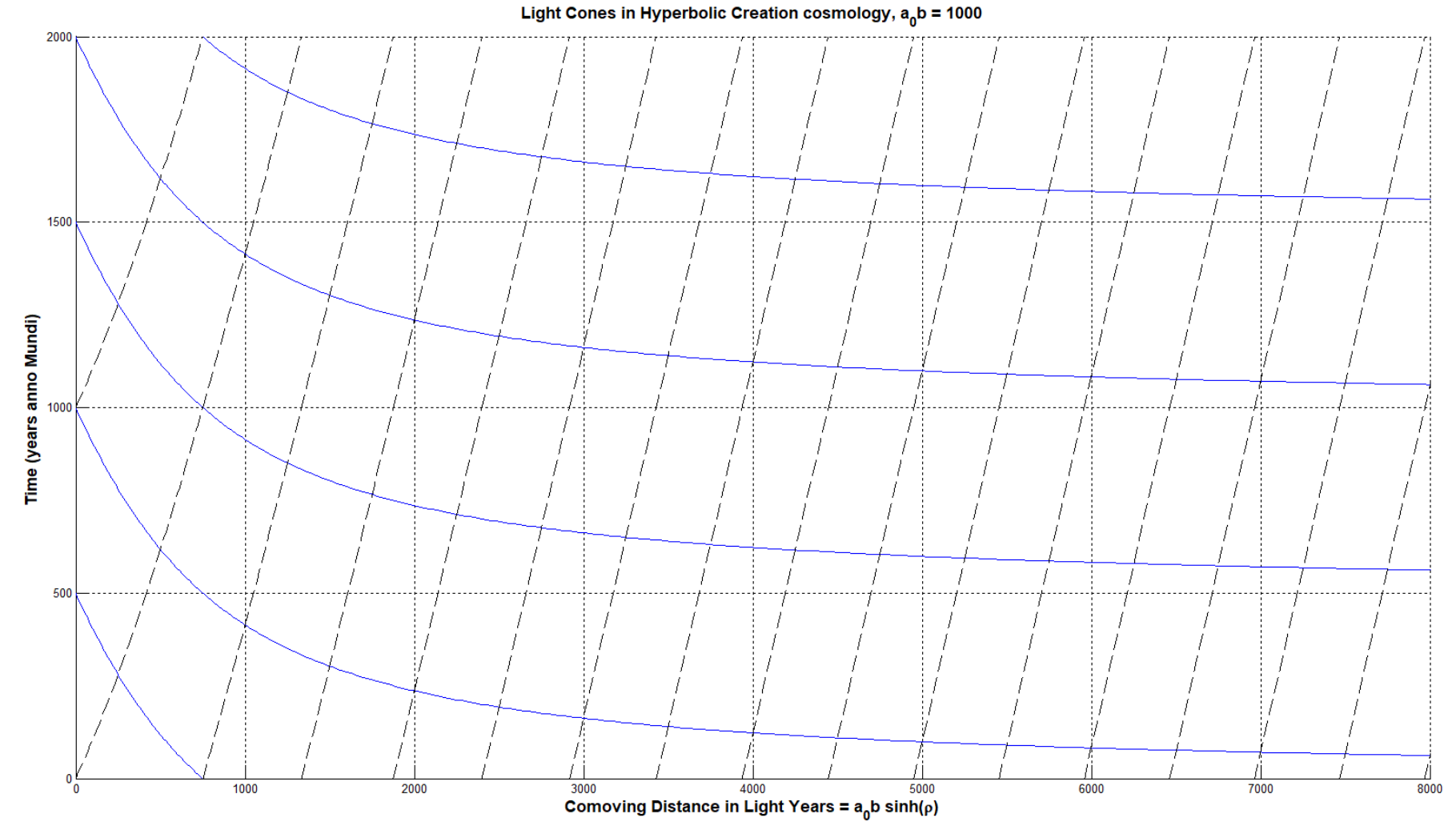

Figure 10. Null Cones in a Hyperbolic YEC Cosmology. The horizontal axis is the distance from the earth whose worldline is the time axis on the left. Solid lines indicate incoming light. Dashed lines are the outgoing light. Note that the global speed of incoming light approaches infinity as distance increases. Conversely the global speed of outgoing light decreases with distance. Since the model is a solution of the EFE, the local speed of light is constant in accordance with the principle of relativity. This graph is drawn at "epoch' and used a value of $a_{0} b=1000$ years for the "temporal radius" of the hyperbolic surfaces of simultaneity. Recall that the parameter $b$ specifies the length of time in which an infinitely distant object crosses the particle horizon. This can be seen in the graph as the incoming light cone with the vertex at 1000 years is asymptotically horizontal to $t=0$ as $\rho$ approaches $\infty$. 
initial creation surface consistent with the biblical revelation.

The main features of the model presented are (1) its foundation on a philosophically and biblically consistent interpretation of the temporal aspects of relativistic physics. The model is based on presentism, not the eternalist view of time. (2) Recognition that the relativity principle says nothing about the existence of a universal cosmic time. Such a cosmic time is consistent with the inability to globally synchronize moving clocks (as in SR) and (in light of the freedom of motion by way of non-gravitational forces) with the inability to operationally detect, by way of local measurements, the value of such a global cosmic time. (3) The model otherwise utilizes the conventional mathematical structure of GR. And, as mentioned, the solution presented herein is a submanifold of the FLRW metric; and, by way of diffeomorphism invariance, it will thus reproduce the observational features of GR cosmological models (such as the FLRW metrics, for example). The only difference between the YEC space-time manifold and the FLRW space-time is the specification of the local time to the initial creation surface. It is that initial condition time function which specifies the YEC sub-manifold of the FLRW by way of the creation surface of simultaneity. And, as we have stated, GR can say nothing about which initial condition is to be accepted. The decision to accept one over another is motivated by philosophical and theological presuppositions alone.

\section{ACKNOWLEDGEMENTS}

Very special thanks to Robert E. Walsh for reading an early version of the paper and for his useful recommendations for improving its clarity. The author is grateful to the reviewers whose constructive comments have lead to several additions and explanations that hopefully add clarity to the paper. Any remaining lack of clarity is entirely the fault of the author.

\section{REFERENCES}

Bondi, H. 1947. Spherically symmetrical models in general relativity. Monthly Notices of the Royal Astronomical Society 107, no. 5,6: 410425 .

Bonnor, W.B. 1956. The formation of nebulae. Zeitschrift für Astrophysik 39:143-159.

Calaprice, A., ed. 2000. The Expanded Quotable Einstein. Princeton: Princeton University Press.

Clowes, R.G., K.A. Harris, S. Raghunathan, L.E. Campusano, I.K. Söchting, and M.J. Graham. 2013. A structure in the early universe at $\mathrm{z} \sim 1.3$ that exceeds the homogeneity scale of the R-W concordance cosmology. Monthly Notices of the Royal Astronomical Society 429, Issue 4:2910-2916. https://doi.org/10.1093/mnras/sts497.

DeFacio, B., P.W. Dennis, and D.G. Retzloff. 1978. Presymmetry of classical relativistic particles. Physical Review D 18:2813-2819.

DeFacio, B., P.W. Dennis, and D.G. Retzloff. 1979. Equivalence of two approaches to noninertial Observers. Physical Review D 20:570-571.

Dingle, H. 1933. On isotropic models of the universe, with special reference to the stability of the homogeneous and static states. Monthly Notices of the Royal Astronomical Society 94:134-158.

Eddington, A.S. 1935. The Nature of the Physical World. London: Dent.

Einstein, A. 1994. Ideas and Opinions [Modern Library edition]. New
York: Random House.

Ellis, G. 2012. Space Time and the Passage of Time. https://arxiv.org/ abs/1208.2611

Enqvist, K. 2008. Lemaitre-Tolman-Bondi model and accelerating expansion. General Relativity and Gravitation 40:451-466.

Faulkner, D.R. 2013. A proposal for a new solution to the light travel time problem. Answers Research Journal 6:279-284.

Frolov, V., and I. Novikov. 1998. Black Hole Physics, Dordrecht: Kluwer Academic Publishers.

Gourgoulhon, E. 2012. 3+1 Formalism in General Relativity, SpringerVerlag.

Hawking, S.W., and G.F.R Ellis. 1973. The Large Scale Structure of Space-time, Cambridge: Cambridge University Press.

Hellaby, C. 1987. A Kruskal-like model with finite density. Classical and Quantum Gravity. 4:635-650.

Hellaby, C., and K. Lake. 1985. Shell crossings and the Tolman model. The Astrophysical Journal 290:381-387. (correction in 1986. The Astrophysical Journal. 300:461).

Kant, Immanuel. 1787. Critique of Pure Reason. $2^{\text {nd }}$ edition [trans. P. Guyer and A.W. Wood, 1998]. Cambridge: Cambridge University Press.

Krasiński, A. 1997. Inhomogeneous Cosmological Models. Cambridge: Cambridge University Press.

Misner, C., K.S. Thorne, and J. Wheeler. 1973. Gravitation. San Francisco: W.H. Freeman.

Novikov, I.D. 1963. On the evolution of a semiclosed world. Astronomicheskii Zhurnal 40:772 [English translation. 1964. Soviet Astronomy. A.J. 7:587].

Novikov, I.D. 2001. R- and T-regions in space-time with spherically symmetric space. General Relativity and Gravitation 33, no. 12, 22592295.

Plebański, J., and A. Krasiński. 2006. An Introduction to General Relativity and Cosmology. Cambridge: Cambridge University Press.

Putnam, H. 1995. Time and physical geometry. In Mathematics, Matter and Method, Philosophical Papers, vol. I., 2nd edition, pp. 198-205. Cambridge: Cambridge University Press.

Reichenbach, H. 1956. The Direction of Time. Berkeley, Los Angeles: University of California Press.

Sussman, R. 1985. Conformal structure of a Schwarzschild black hole immersed in a Friedman universe. General Relativity and Gravitation. 17, no. 3:251-291.

Synge, J.L. 1971. Relativity: The General Theory. Amsterdam: NorthHolland Publishing Company.

Tolman, R.C. 1934. Effect of inhomogeneity on cosmological models. Proceedings of the National Academy of Sciences of the United States of America 20:169-176.

Unger, R., and L. Smolin. 2015. The Singular Universe and the Reality of Time. Cambridge: Cambridge University Press.

Weyl, H. 1949. Philosophy of Mathematics and Natural Science. Princeton: Princeton University Press. 
Whitrow, G.J. 1980. The Natural Philosophy of Time, $2^{\text {nd }}$ edition. Oxford: Oxford University Press.

Yadav, J.K., J.S. Bagla, and N. Khandai. 2010. Fractal dimension as a measure of the scale of homogeneity. Monthly Notices of the Royal Astronomical Society 405:2009-2015.

Zel'dovich, la.B., and L.P. Grishchuk. 1984. Structure and future of the 'new' universe. Monthly Notices of the Royal Astronomical Society 207:23-28.

\section{THE AUTHOR}

Dr. Dennis obtained his Ph.D. in physics from the University of Missouri. His areas of expertise are gauge field theories with spontaneous symmetry breaking; quantum field theory; and general relativity. He is the author of papers in the areas of quantum field theory; and invariant methods in special and general relativity. Over the years, Dr. Dennis worked on projects in the areas of physical oceanography, algorithms for the Hubble Space Telescope, tracking algorithms, and other mathematical algorithms; been awarded three patents, has been recognized by awards from NASA, TRW, and Litton, and is a member of the American Physical Society.

\section{APPENDIX A: PUTNAM ON SPECIAL RELATIVITY} AND ETERNALISM.

In this appendix we critique Hilary Putnam's (Putnam, 1995) argument for "eternalism."

Putnam's argument is based on the conventional definition of "simultaneity" used in Special Relativity (SR). The purely formal definition of simultaneity leads to the false conclusion of Putnam $\&$ Lewis and eternalism. The argument endows the surfaces of simultaneity (specified by a constant time coordinate in an inertial reference frame) with a metaphysical reality. In fact, Putnam endows every surface of simultaneity for all inertial observers with a reality. That assumption is the core of his rejection of presentism. This argument is thus based on a mistaken notion of the metaphysical import of the conventional nature of simultaneity in SR along with a non-rigorous application of the use of coordinates in the theory of manifolds.

First, the concept of simultaneity in SR is certainly not a welldefined concept. In fact, the argument that SR defeats presentism can be distilled down to a reliance on the merely operational definition that a spacelike surface with a Minkowski coordinate $t=$ constant , in a given inertial reference frame, defines simultaneity, i.e. the set of events that are "simultaneous" with the observer. The fact that time coordinates are not invariant under a general Lorentz transformation is, of course, a fundamental core of SR. From the conventional definition of simultaneity, it follows, then, that there can be no one universal $t=$ constant surface. But the operational method of defining times for remote events only results in mere coordinates. Coordinates, or ways of labeling events, have no necessary connection with the reality of events. A simple counter example suffices. I can certainly, sans relativity, talk about the time coordinate for future events, within presentism. If today is noon on Monday the $21^{\text {st }}$ of April, I can speak of an appointment, 24 hours in the future, on Tuesday the $22^{\text {nd }}$ of April at noon. Such a coordinate label does not thereby make my appointment real at the present. The point is simply that an operational assignment of a coordinate value to an event does not necessarily endow it with metaphysical significance.

This point should be entirely noncontroversial. It is a recognized fact of manifolds that the properties of the manifold are independent of the coordinate mesh used to label points. The coordinates are entirely arbitrary. In fact, it is in general necessary to cover manifolds with multiple overlapping mappings of coordinates known as charts. This is required as many manifolds are not homeomorphic to an n-dimensional Euclidean space, $R^{n}$.

The most ubiquitous example of non-Euclidean coordinates is polar coordinates for the standard two-dimensional plane, $R^{2}$. Another common example are coordinates on the two-dimensional sphere, $S^{2}$, which require more than one chart to cover the surface of the sphere. (Consider transverse Mercator coordinates, and polar stereographic coordinates, both of which require at least two charts.) One must be careful drawing conclusions from mere coordinates. The coordinate independent properties of manifolds are represented by invariants built from tensors. Such invariants (or other geometrical properties that are independent of coordinates) should be used when deducing properties of solutions in SR and GR.

Putnam's argument boils down to the following. (See Figure 11). Putnam considers two observers, "me" and "you." You are moving in the negative direction relative to me. Thus, by the Lorentz transformation "my now" $(t=0)$ differs from "your now" $\left(t^{\prime}=0\right)$. Next Putnam asserts that "my now" is "real" to me, or that all things that are "simultaneous," according to the Lorentz transformation, with "my now" must likewise be "real" to me, i.e. existing at the same moment. This assumption is dubious and, in fact, already presupposes eternalism (it thus begs the question). Putnam next asserts that all the events with coordinates $t=$ constant are "real" to me and, likewise, all the events with $t^{\prime}=$ constant must be "real" to you. This follows from the absence of preferred frames of reference. Finally, since "you" and "me" are coincident at $t=t^{\prime}=0$, we are both "real" to each other. It then follows by transitivity that if all events in the hyperspace labeled "you now" are real, and "you" are real to "me" at $t=0$ and all events in "my now" are real to me, then all the events in "your now" are equally real to you, and therefore, real to me also and to all the events in "my now." Thus, "my now" and "your now" are equally real. This conclusion is eternalism in a nutshell. All events in the space-time manifold, past, present and future, are equally "real" to you and me, and equally real for any, and all, observers. Thus, time is an illusion.

The following observations are relevant to rebutting Putnam's arguments:

(1) For any observer, the $t=$ constant surface is never actually observed - ever! Observers only see events causally connected to the current time by way of the past null cone.

(2) The fact that there are different time labels for a space-time event in no way proves there is not a real present. It only implies that such an objective now is not determinable for us - not that it does not exist!

(3) Proper time for all observers is not an exact differential (cf. equation (2) above). The methodological process of extending 


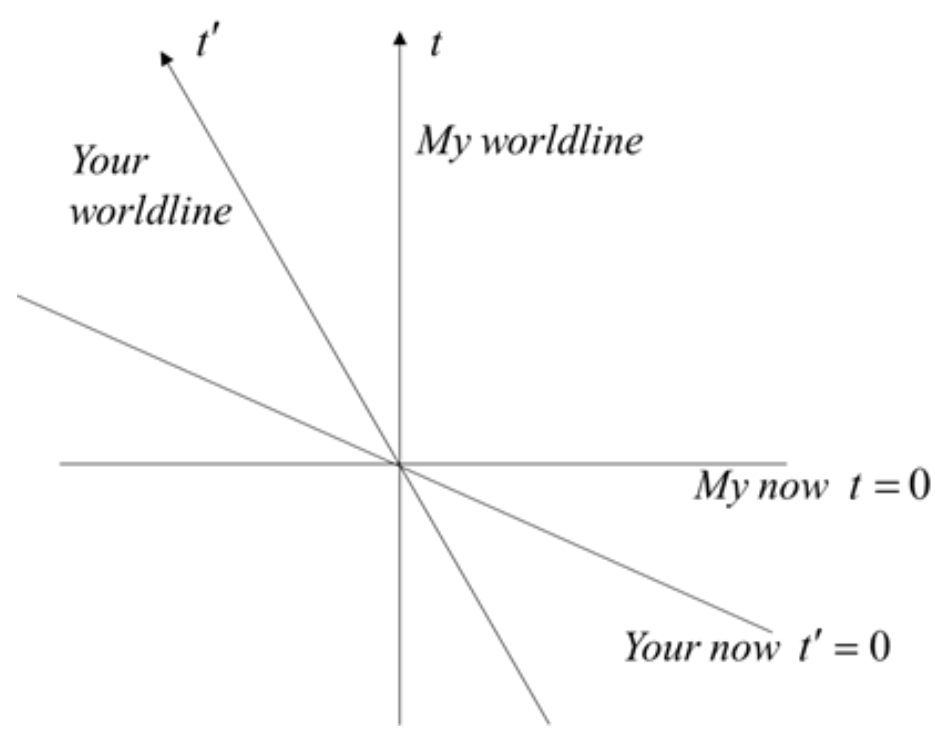

Figure 11. Space-time diagram used in Putnam's argument for Eternalism. You are traveling relative to me in the negative $x$ direction. By the Lorentz transformation and the Einstein definition of "simultaneity" we have different sections of space-time that we call "now." Putnam asserts that all events "simultaneous" with an observer are "real." At time $t=t^{\prime}=0$ we are both at the origin and thus equally real to each other. By transitivity Putnam then argues that (1) "my now" is real to me, (2) you are real to me, (3) "your now" is real to you, therefore (4) "my now" and "your now" are equally "real" in a timeless cosmos.

an observer's local proper time to a global time coordinate is necessarily observer dependent - but such a process has nothing to say metaphysically about presentism. This extension of proper time is a procedure for consistently labeling locally space-time events, and thereby in SR providing a foliation based on nothing more than a conceptual parallel transport of an inertial observer's 4-velocity that creates a surface-orthogonal vector field. The author (with DeFacio and Retzloff) used such an approach (via invariant methods) to derive exact equations of motions for a particle observed by arbitrarily accelerating observers. The analysis included equations for both space-like and null-cone foliations. For details see DeFacio, Dennis, and Retzloff, (1978, 1979).

(4) Finally, asserting that a constant time surface according to the
Lorentz transformation is "real" begs the question. We show below that asserting the "reality" of events in an artificial $t=$ constant hypersurface leads to absurd conclusions. We will see that such an assumption leads to the conclusion that some events become "unreal" since they are not assigned a time.

So then, a simple consideration of the absurdities that ensue in the case of accelerated observers from the SR definition of "now" is sufficient to rebut the eternalist view. One interesting case that clearly demolishes any metaphysical connection of coordinates to simultaneity or of the reality of events is the case of an inertial observer who suffers an impulsive acceleration changing his speed from zero to speed $v$ relative to an inertial frame $S$. (See Figure 12 below).

Using the Lorentzian operational definition of simultaneity, we see that the event $\mathrm{E}$, which was in the future a moment before the impulse, instantaneously "jumps" into the past a moment after the impulse. It should be noted that events in the negative $\mathrm{x}$ direction also jump into the past instantaneously and will in fact be simultaneous again in the "future." Another anomaly and the most egregious is that the event $\mathrm{E}$ is never simultaneous with any event in the $S^{\prime}$ frame. Thereby rendering it "unreal" by Putnam. It is most odd that an observer undergoing arbitrary local time dependent accelerations can instantaneously make entire regions of remote events unreal. This underscores the artificiality of Putnam's analysis and is yet another example in which the historical development and terminology of SR leads to misunderstanding and a fallacious conclusion. Unfortunately, such confusions are still among trained physicists.

A naïve application of the definition of simultaneity as the hypersurface $t^{\prime}=0$ by way of a Lorentz transformation would lead one to the conclusion that remote events in the region labeled $X^{-}$ instantaneously jumped back into the past due to the acceleration of the observer at event $X^{+}$. Likewise, events in region $X^{+}$are now no longer simultaneous with $O$.

This uncritical application of Einstein-Poincare simultaneity yields a very bizarre instance of action-at-a- distance time travel! These conclusions are absurd and rest upon (1) an arbitrary definition of simultaneitv and endowing that propertv definition with reality;

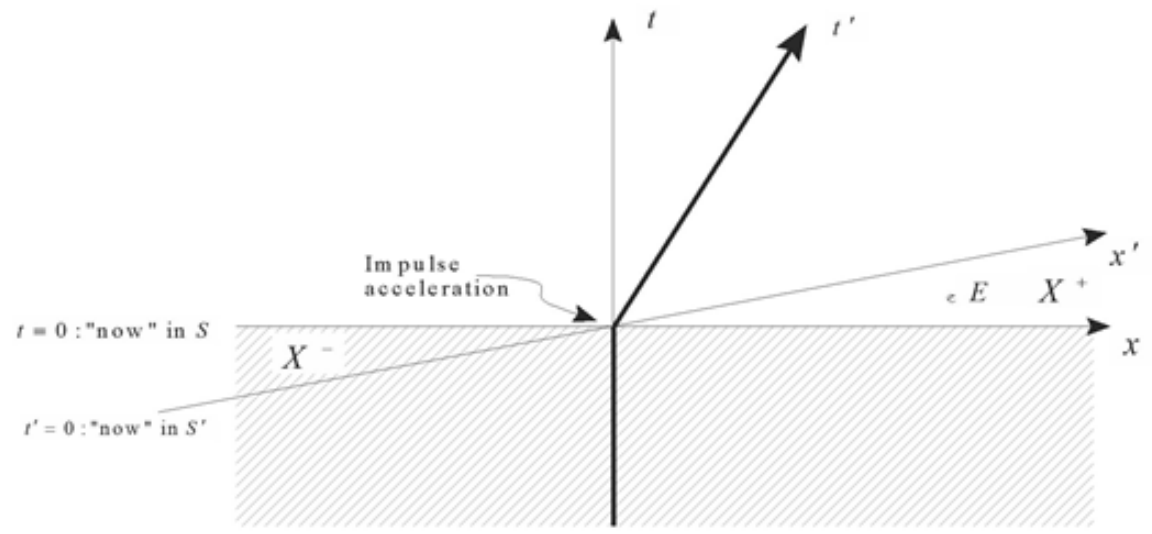

Figure 12. An observer $S^{\prime}$ undergoing an impulse acceleration resulting in a change of velocity at time $t=0$. After the boost, the observer in the primed frame of reference is in an inertial frame moving at velocity $v$ in the positive x-direction of observer $S$. A naïve application of the Lorentz transformation (LT) to observer $S^{\prime}$ and its extension into regions where the assumptions used in the derivation of the LT do not apply lead to manifestly absurd conclusions as described in the text. 
and (2) a misapplication of the relativity principle in uncritically applying the Lorentz transformation after $t=0$, rather than deriving a consistent set of coordinates based on the radar-ranging method and based on the first principle of special relativity, viz., the constancy of the speed of light independent of the observer's speed. Recall that the Lorentz transformation was derived on the assumption of two different inertial observers in uniform relative motion; therefore, any uncritical application of the transformation to accelerated observers is invalid.

The following derivation of continuous time coordinates based on "radar-ranging" is not essential to the main purpose of this paper but is provided as an example of a consistent and operational construction of coordinates based on the fundamental physics of the principle of relativity. In this regard, it is the same theoretical procedure that Einstein used to construct the coordinates in the case of globally inertial observers; and illustrates how to avoid the pitfalls of naively applying Lorentz transformations to cases in which they are inapplicable.

A correct method is to use radar-ranging. Refer to Figure 13. In the radar-ranging method, a light pulse is transmitted by the observer in frame $S^{\prime}$ at time $t_{A}$, subsequently reflected from an object at $(x, t)$ and then received at time $t_{B}$. (It should be noted that the artificial definition of simultaneity based on the LT results in the assignment of time coordinates to events that have not yet been observed at time $t=0$. It is thus at odds with the very operational construction of SR itself. We note that when using the radar- ranging method -a fully operationalist construction - coordinates are always assigned to events that lie in the observer's past; and thus, have already occurred. It is necessarily compatible with the reality of time and its flow. This method is thus fully causal and results in causally consistent coordinates that can be used for computing physical quantities.)

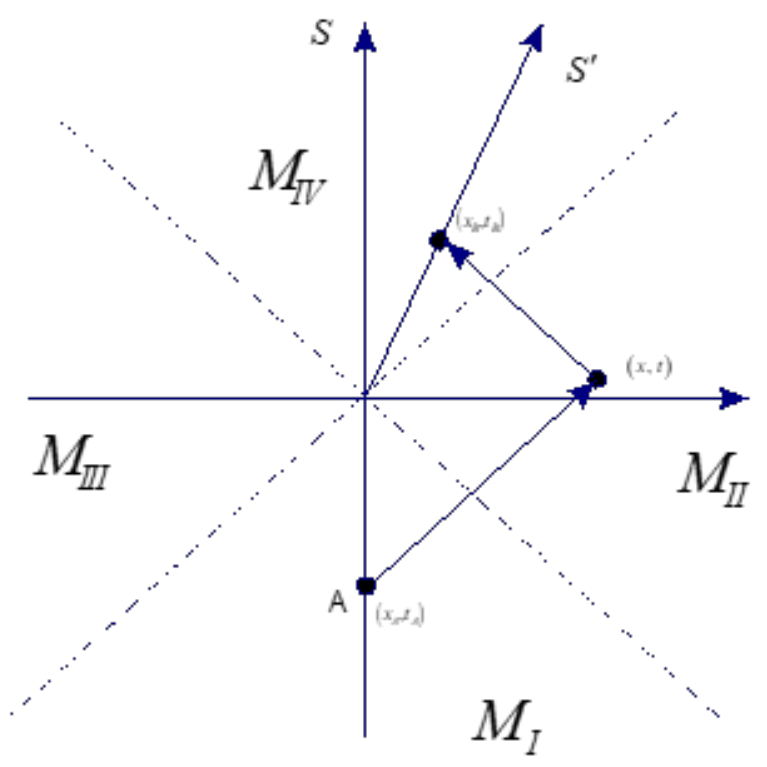

Figure 13. Radar-ranging method for the frame $S^{\prime}$. The observer is not globally inertial, but is inertial before and after $t=0$. Coordinates are assigned by sending a light signal to a distant event and receiving the returned signal. Using the constancy of the speed of light, consistent coordinates can be assigned that are physically based on the fundamental principle of Einstein's principle of relativity.
So, we consider an observer who undergoes an instantaneous velocity increase at time $t=0$. (See Figure 12) Before $t=0, S^{\prime}$ is an inertial frame coincident with $S$. After $t=0, S^{\prime}$ is in an inertial frame with velocity $v$ in the $\mathrm{x}$-direction relative to $S$.

The radar-ranging method consists of the observer emitting a light pulse at time $t_{A}^{\prime}$ to a target at a location a distance $t_{B}^{\prime}$ and measuring the time of observation of the reflected signal at time $t_{B}^{\prime}$ . Since the speed of light is invariant we can compute the time of reflection based on the clock readings $t_{B}^{\prime}, t_{A}^{\prime}$ in frame $S^{\prime}$ as:

$t^{\prime}=\frac{1}{2}\left(t_{B}^{\prime}+t_{A}^{\prime}\right)$

The range of the object measured by $S^{\prime}$ is then given by:

$r^{\prime}=\frac{1}{2}\left(t_{B}^{\prime}-t_{A}^{\prime}\right)$

We divide Minkowski space into four coordinate charts, labeled $M_{I}, M_{I I}, M_{I I I}, M_{I V}$. These are selected based on the speed of $S^{\prime}$ at the time of transmission and reception. In $M_{I}$, transmission and reception occur when $S$ is at rest relative to $S$. Thus, for all events in $M_{I}, S$ and $S^{\prime}$ agree as to assigned coordinates:

$t^{\prime}=t$

$x^{\prime}=x$

In $M_{I V}$, transmission and reception occur when $S^{\prime}$ is moving at

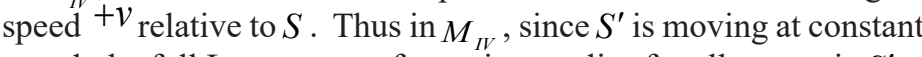
speed, the full Lorentz transformation applies for all events in $S^{\prime}$ :

$$
\begin{aligned}
& t^{\prime}=\frac{t-v x}{\sqrt{1-v^{2}}} \\
& x^{\prime}=\frac{x-v t}{\sqrt{1-v^{2}}}
\end{aligned}
$$

In $M_{I I}$ and $M_{I I I}$, however, transmission occurs when $S^{\prime}$ is at rest relative to $S$, and reception occurs when $S^{\prime}$ is moving at speed $S^{\prime}$ relative to $S$. Thus in $M_{I I I}$ and $M_{I I I}$ the Lorentz transform does not apply and the transformation between the reference frames must be derived from first principles. Finally, note that, in $M_{I I}$ and $M_{I I I}$ the $x$ coordinate is positive or negative, respectively, for the radar method. In $M_{I I}$ the $x$ coordinate is the same as range, while in $M_{I I I}$ the $\mathrm{x}$-coordinate is the negative of the range.

We now derive the coordinate charts for $M_{I I}$ and $M_{I I}$, using the constancy of the speed of light and the time dilation of $S^{\prime}$ clock in region $M_{I V}$.

In region $M_{I I}$, writing the kinematic equations for the null rays, gives:

$t_{B}-t=x-v t_{B}$

$t-t_{A}=x$

or

$t_{B}=\frac{x+t}{1+v}$

$t_{A}=t-x$

The radar range equations yield: 
$t^{\prime}=\frac{1}{2}\left(t_{A}^{\prime}+t_{B}^{\prime}\right)=\frac{1}{2}\left(t_{A}+t_{B} \sqrt{1-v^{2}}\right)$

$x^{\prime}=\frac{1}{2}\left(t_{B} \sqrt{1-v^{2}}-t_{A}\right)$

Substituting the times from the null ray solutions gives:

$$
\begin{aligned}
t^{\prime} & =\frac{1}{2}\left(t_{A}^{\prime}+t_{B}^{\prime}\right) \\
& =\frac{1}{2}\left(t-x+(x+t) \sqrt{\frac{1-v}{1+v}}\right) \\
& =\frac{1}{2}\left[\left(\sqrt{\frac{1-v}{1+v}}+1\right) t+\left(\sqrt{\frac{1-v}{1+v}}-1\right) x\right] \\
x^{\prime} & =\frac{1}{2}\left((x+t) \sqrt{\frac{1-v}{1+v}}-(t-x)\right) \\
& =\frac{1}{2}\left[\left(\sqrt{\frac{1-v}{1+v}}-1\right) t+\left(\sqrt{\frac{1-v}{1+v}}+1\right) x\right]
\end{aligned}
$$

$\operatorname{In}_{M_{I I I}}$ the null rays satisfy:

$t_{B}-t=v t_{B}-x$

or $t_{A}=-x$

$t_{B}=\frac{t-x}{1-v}$

$t_{A}=t+x$

Therefore

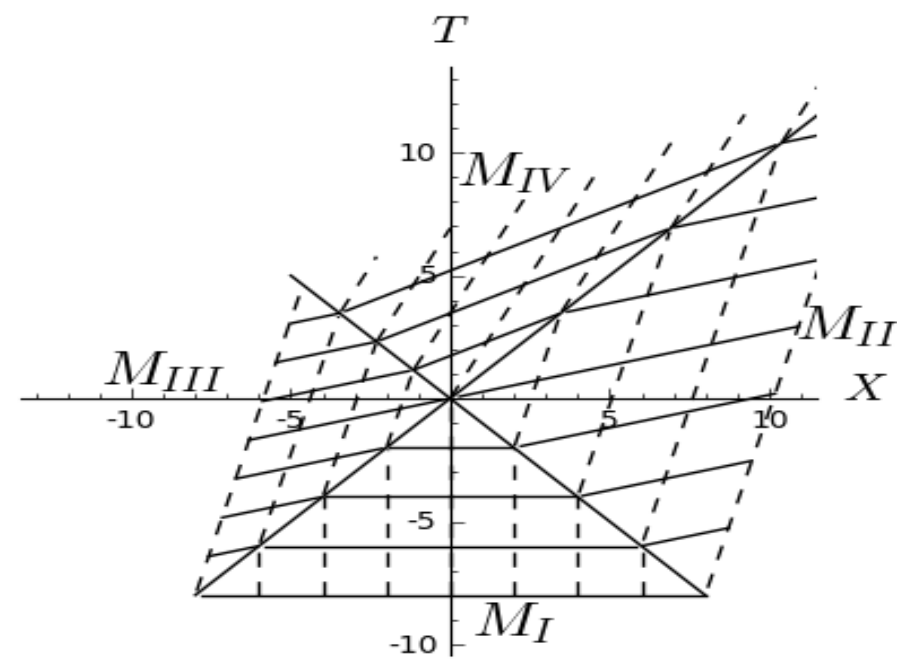

Figure 14. Radar range coordinates for an observer undergoing an impulse acceleration. This is a global and continuous coordinate system derived from first principles of relativity. Every event in the space-time necessarily has been observed before coordinates are assigned.

$$
\begin{aligned}
t^{\prime} & =\frac{1}{2}\left(t_{A}^{\prime}+t_{B}^{\prime}\right)=\frac{1}{2}\left(t_{A}+t_{B} \sqrt{1-v^{2}}\right) \\
x^{\prime} & =-\frac{1}{2}\left(t_{B} \sqrt{1-v^{2}}-t_{A}\right) \\
t^{\prime} & =\frac{1}{2}\left(t_{A}^{\prime}+t_{B}^{\prime}\right) \\
& =\frac{1}{2}\left(t+x+(t-x) \sqrt{\frac{1+v}{1-v}}\right) \\
& =\frac{1}{2}\left[\left(\sqrt{\left.\frac{1+v}{1-v}+1\right)}\right) t-\left(\sqrt{\frac{1+v}{1-v}}-1\right) x\right] \\
x^{\prime} & =-\frac{1}{2}\left(t_{B} \sqrt{1-v^{2}}-t_{A}\right) \\
= & -\frac{1}{2}\left((t-x) \sqrt{\frac{1+v}{1-v}}-(t+x)\right) \\
= & \frac{1}{2}\left[\left(\sqrt{\frac{1+v}{1-v}}+1\right) t-\left(\sqrt{\frac{1+v}{1-v}}-1\right) x\right]
\end{aligned}
$$

The resulting coordinate lines for the moving observer are shown in Figure 14. The continuity of the coordinates in both space and time and the absence of coordinate anomalies is clear. It is a global and continuous coordinate system derived from first principles of relativity. Every event in the space-time to which coordinates are assigned have necessarily been observed since the procedure is based on the operational requirements of the principle of relativity.

\section{APPENDIX B: JOINING EQUATIONS FOR THE INHOMOGENEOUS "BAR BELL" COSMOLOGY.}

We will construct the bar bell cosmology from two FLRW regions (labeled "1" and "2") and a Schwarzschild region labeled "3." We will use comoving coordinates for constructing a global radial coordinate $r$. We will take our cue for this coordinate by using the embedding diagram and noting that the Novikov coordinate uses the maximum radial coordinate of a freely falling particle as the constant comoving coordinate of the particle. We will use that feature for the FLRW to convert the usual radial "angular" FLRW coordinate to a "linear" radial coordinate. Figure 15 displays the pertinent variables and their relations. The Schwarzschild region lies in the range of Novikov coordinates: $r_{1} \leq r \leq r_{2}$.

\section{Embedding Maps}

To construct a mathematically precise solution we will need to specify the details of the Schwarzschild region mentioned above. To do this we will construct the embedding of a $t=$ constant and $\theta=\pi / 2$ two-dimensional cross section of the Schwarzschild geometry with surface metric:

$d s^{2}=\left(1-\frac{2 M}{r}\right)^{-1} d r^{2}+r^{2} d \varphi^{2}$

To embed this in three-dimensional Euclidean space with metric in cylindrical coordinates:

$d s^{2}=d z^{2}+d r^{2}+r^{2} d \varphi^{2}$

one writes $z$ as a function of $r$. 
Thus obtaining

$d s^{2}=\left(1-\frac{2 M}{r}\right)^{-1} d r^{2}+r^{2} d \varphi^{2}=\left[1+\left(\frac{d z}{d r}\right)^{2}\right] d r^{2}+r^{2} d \varphi^{2}$

With solution:

$r=2 M+\frac{\left(z-z_{0}\right)^{2}}{8 M}$

We have introduced the constant $z_{0}$ which specifies the center of symmetry for later convenience. In anticipation of later analysis, we change the variables as follows:

$r \rightarrow R$

$z \rightarrow r$

so that the embedding is specified as:

$R=2 M+\frac{\left(r-r_{0}\right)^{2}}{8 M}$

Now $r$ is no longer the Schwarzschild radial coordinate ("curvature coordinate") but is the Novikov comoving radial coordinate. The function $R$, which corresponds to the Schwarzschild radius, is now a function of $r$ and is the radius of the spherical shell at Novikov radius $r$. We now discuss the Novikov coordinates for the Schwarzschild solution.

\section{Novikov Coordinates}

We will also find it useful to utilize a version of Novikov coordinates for the maximal Schwarzschild manifold (Misner et al. 1973, pp. 826-7). Our path to a YEC cosmology will be via stitching together subsets of various space-times. One of these space-times will be the FLRW space-times which consists of freely falling matter (hence inertial) and which utilize "comoving" coordinates. Comoving coordinates have a great interpretational advantage since the coordinates are based directly on physical principles. Also, since Novikov coordinates are an example of comoving coordinates for the Schwarzschild geometry, they will be useful for facilitating the joining conditions between the solutions. When we do this, all coordinates are comoving and the time coordinate becomes the proper time of all freely falling observers in the cosmological solution. The joining conditions are easier because the L-T class of spherically symmetric inhomogeneous solutions all use a global comoving coordinate system.

In a comoving coordinate system each comoving observer is freely falling and assigns his elapsed proper time as the time coordinate for each event along his world line. For these coordinate systems the $g_{t t}$ component of the metric tensor is necessarily -1 . The spatial coordinates are constant for each observer and consist of the spherical coordinates $(\theta, \varphi)$ and (a function of) the radial coordinate $R$ which labels the observer's initial position. As the observer free-falls in the gravitational field he follows a geodesic. That geodesic is uniquely determined by the initial point and the fourvelocity. Each event along the geodesic is thus determined by the "arc-length" (i.e. proper time $t$ ) along the geodesic and the initial position $R, \theta$ and $\varphi$. The set of observers for Novikov coordinates consists of observers falling from rest at distinct "distances" $R$ from an arbitrarily chosen instant of time. A convenient choice is to assume the clocks are synchronized at the instant of maximal radius.

Novikov originally used a dimensionless coordinate related to the Schwarzschild $R$ (recall our change of coordinate labels above) coordinate by:

$r=\sqrt{\frac{R}{2 M}-1}$

For our analysis we will use a dimensional Novikov radial coordinate defined as:

$r=r_{0}+4 M \sqrt{\frac{R}{2 M}-1}$

In this case our metric, in the notation of the L-T solutions (cf. equation (5)), becomes:

$d s^{2}=-d t^{2}+\frac{\left(R^{\prime}\right)^{2}}{1+2 E(r)} d r^{2}+R^{2} d \Omega^{2}$

(where we now omit the asterisk and, as mentioned previously, set the radius function as $R(t, r)$ rather than $r)$. The function $E(r)$, which is a measure of the free-falling particle's energy, will be discussed below when we examine time-dependent spherically symmetric space-times of the Lemaître, Tolman, Bondi (L-T) family.

In each of the FLRW regions the solution is:

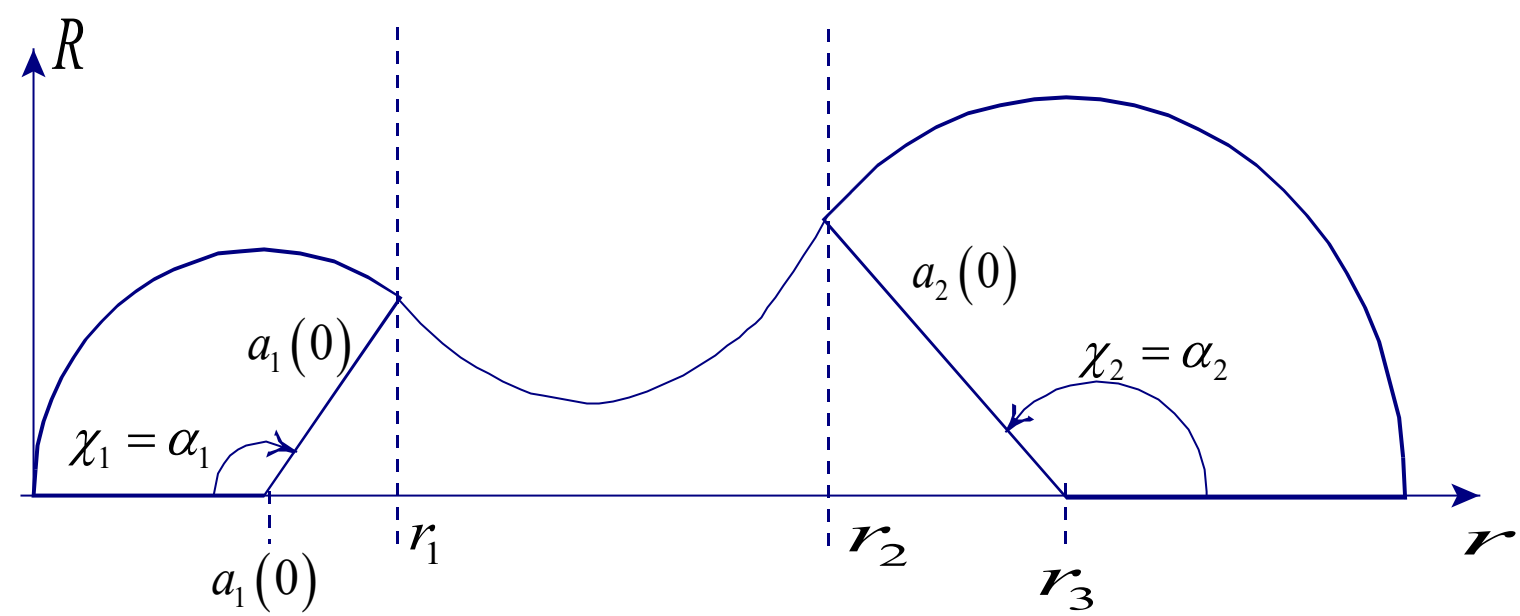

Figure 15. Profile of embedding diagram of "bar bell" cosmology. Construction of global radial comoving coordinate " $r$ " 
$d s^{2}=-d t_{1}^{2}+a_{1}^{2}(t)\left(d \chi_{1}^{2}+\sin ^{2} \chi_{1} d \Omega^{2}\right)$

$d s^{2}=-d t_{2}^{2}+a_{2}^{2}(t)\left(d \chi_{2}^{2}+\sin ^{2} \chi_{2} d \Omega^{2}\right)$

The "angular" radial coordinates $\chi$ are measured from the two poles of the closed cosmology. The angles $\alpha_{2}$ and $\alpha_{2}$ correspond to linear coordinates $r_{1}$ and $r_{2}$, respectively.

The parametric solutions for $R_{k}$ and $t_{k}$ in terms of the "cycloidal" parameter $\eta$ are:

$R_{k}\left(\eta, \chi_{k}\right)=\frac{a_{k}(0) \sin \chi_{k}}{2}(1+\cos \eta)$

$t_{k}(\eta)=\frac{a_{k}(0)}{2}(\eta+\sin \eta)$

$a_{k}(0)$ for $k=1,2$ is the maximum radius of the regions 1 and 2 at maximum expansion at $\eta=0$. The parameter $\eta$ ranges over $[-\pi, \pi]$. The big bang occurs at $\eta=-\pi$ and the big crunch at $\eta=\pi$.

The above solution is obtained from the general L-T solutions:

$R_{k}\left(\eta, \chi_{k}\right)=\frac{M_{k}\left(\chi_{k}\right)}{-2 E_{k}\left(\chi_{k}\right)}(1+\cos \eta)$

$t_{k}\left(\eta, \chi_{k}\right)=\frac{M_{k}\left(\chi_{k}\right)}{2\left(-E_{k}\left(\chi_{k}\right)\right)^{3 / 2}}(\eta+\sin \eta)$

The function $M$ and $E$ are given by:

$M_{k}\left(\chi_{k}\right)=a_{k}(0) \sin ^{3} \chi_{k}$

$E_{k}\left(\chi_{k}\right)=-\frac{1}{2} \sin ^{2} \chi_{k}$

The joining of the three regions is performed by requiring continuity of the functions $M$ and $E$. Clearly the function $M$ is continuous if there are no delta-function surface layers, which is the case for our model.

A property of the closed universe is that the total gravitational mass is zero. Thus

$M_{\text {total }}=\frac{4 \pi}{3} \int_{0}^{r_{\max }} \rho(0, r) \frac{d}{d r}\left[R^{3}\right] d r=0$

In the Schwarzschild region $\rho=0$; therefore the only contributions to $M_{\text {total }}$ are in the FLRW regions

$$
\begin{aligned}
0 & =\frac{4 \pi}{3} \int_{0}^{r_{1}} \rho_{1}(0) \frac{d}{d r}\left[R^{3}\right] d r+\frac{4 \pi}{3} \int_{r_{2}}^{r_{\max }} \rho_{2}(0) \frac{d}{d r}\left[R^{3}\right] d r \\
& =\frac{4 \pi}{3}\left[\rho_{1}(0) R^{3}\left(0, r_{1}\right)-\rho_{2}(0) R^{3}\left(0, r_{2}\right)\right]
\end{aligned}
$$

Therefore, $M\left(\alpha_{1}\right)=M\left(\alpha_{2}\right)=r_{g} / 2$ where $r_{g}=2 M$ is the "gravitational" radius in the Schwarzschild region.

Thus

$M\left(\alpha_{1}\right)=a_{1}(0) \sin ^{3} \alpha_{1}=M\left(\alpha_{2}\right)=a_{2}(0) \sin ^{3} \alpha_{2}=r_{g} / 2$

To relate the angular variable $\chi$ to the linear coordinate $r$, we define the linear radial coordinate of points in the FLRW regions by their projection onto the " $r$ " axis of the embedding space at maximal expansion. At the boundary points $r_{1}$ and $r_{2}$ we then have the following relation between $\chi$ and $r$

$r_{1}=a_{1}(0)\left(1-\cos \alpha_{1}\right)$

$r_{2}=r_{3}+a_{2}(0) \cos \alpha_{2}$

In region 3, we proceed similarly and write the solution in Novikov coordinates:

$d s^{2}=-d t^{2}+\frac{\left(R^{\prime}\right)^{2}}{1+2 E(r)} d r^{2}+R^{2} d \Omega^{2}$

Where the parametric solutions are:

$R(0, r)=r_{g}+\frac{1}{4 r_{g}}\left(r-r_{0}\right)^{2}$

We can now solve for the "gravitational" center of the Schwarzschild region:

$R\left(0, r_{1}\right)=r_{g}+\frac{1}{4 r_{g}}\left(r_{1}-r_{0}\right)^{2}=a_{1}(0) \sin \alpha_{1}$

Therefore

$\left[r_{g}+\frac{1}{4 r_{g}}\left(r_{1}-r_{0}\right)^{2}\right] \sin ^{2} \alpha_{1}=a_{1}(0) \sin ^{3} \alpha_{1}=r_{g}$

And solving for $r_{0}$

$\frac{1}{4 r_{g}}\left(r_{1}-r_{0}\right)^{2} \sin ^{2} \alpha_{1}=r_{g} \cos ^{2} \alpha_{1}$

$r_{0}=r_{1}-2 r_{g} \cot \alpha_{1}$

The matching at the other boundary yields:

$r_{0}=r_{2}-2 r_{g} \cot \alpha_{2}$

Using these relations, we obtain the embedding diagram shown in Figure 5. 